\title{
The Cauchy Problem for a Weakly Dissipative 2-Component Camassa-Holm System
}

\author{
Sen Ming, ${ }^{1}$ Han Yang, ${ }^{1}$ and Yonghong $\mathrm{Wu}^{2}$ \\ ${ }^{1}$ School of Mathematics, Southwest Jiaotong University, Chengdu 610031, China \\ ${ }^{2}$ Department of Mathematics and Statistics, Curtin University, Perth, WA 6845, Australia
}

Correspondence should be addressed to Han Yang; hanyang95@263.net

Received 18 November 2013; Accepted 14 December 2013; Published 3 February 2014

Academic Editor: Shaoyong Lai

Copyright (c) 2014 Sen Ming et al. This is an open access article distributed under the Creative Commons Attribution License, which permits unrestricted use, distribution, and reproduction in any medium, provided the original work is properly cited.

The weakly dissipative 2-component Camassa-Holm system is considered. A local well-posedness for the system in Besov spaces is established by using the Littlewood-Paley theory and a priori estimates for the solutions of transport equation. The wave-breaking mechanisms and the exact blow-up rate of strong solutions to the system are presented. Moreover, a global existence result for strong solutions is derived.

\section{Introduction}

We consider the following weakly dissipative 2-component Camassa-Holm system:

$$
\begin{gathered}
u_{t}-u_{x x t}+2 k u_{x}+3 u u_{x}+\lambda u^{2 n+1} \\
-\beta u^{2 m} u_{x x}+\rho \rho_{x}=2 u_{x} u_{x x}+u u_{x x x}, \\
t>0, x \in \mathbb{R}, \\
\rho_{t}+(\rho u)_{x}=0, \quad t>0, x \in \mathbb{R}, \\
u(x, 0)=u_{0}(x), \quad x \in \mathbb{R}, \\
\rho(x, 0)=\rho_{0}(x), \quad x \in \mathbb{R},
\end{gathered}
$$

where $k, \lambda, \beta \geq 0$ are constants, $n, m$ are natural numbers, $(x, t) \in \mathbb{R} \times \mathbb{R}^{+}$, and $\left(u_{0}, \rho_{0}-1\right) \in B_{p, r}^{s} \times B_{p, r}^{s-1}$ with $s>$ $\max (3 / 2,1+1 / p)$

In system (1), if $\lambda=\beta=\rho=0$, we get the classical Camassa-Holm equation [1]:

$$
u_{t}-u_{x x t}+2 k u_{x}+3 u u_{x}=2 u_{x} u_{x x}+u u_{x x x}
$$

where $u(x, t)$ is the fluid velocity in $x$ direction (or equivalently the height of the water's free surface above a flat bottom) and $k$ is a constant related to critical shallow water wave speed. The alternative derivation of (2) as a model for water waves can be found in Constantin and Lannes [2]. Equation (2) models for the propagation of shallow water waves have attracted attention of many researchers with two remarkable features. The first one is the presence of solutions in the form of peaked solitary waves for $k=0$. The peakon $u(x, t)=c e^{-|x-c t|}$ with $c \neq 0$, which is a feature observed for the traveling waves of largest amplitude [3-6]. The other feature is that the equation has breaking waves. In other words, the solutions remain bounded while their slope becomes unbounded in finite time. For $k>0$, the solitary waves are stable solitons $[3-5,7]$. Li and Olver [8] not only obtained the local posedness but also gave the conditions which could lead to some solutions blowing up in finite time in Sobolev space $H^{s}$ with $s>3 / 2$. For other methods to establish the local well-posedness and global existence of solutions to the Camassa-Holm equation or other shallow water models, the reader is referred to [9-18] and the references therein.

In general, it is difficult to avoid the energy dissipation mechanisms in a real world. Thus, different types of solutions 
for dissipative Camassa-Holm equation have been investigated. For example, Wu and Yin [19] studied the dissipative Camassa-Holm equation:

$$
\begin{array}{r}
u_{t}-u_{x x t}+3 u u_{x}+\lambda\left(u-u_{x x}\right)=2 u_{x} u_{x x}+u u_{x x x}, \\
t>0, x \in \mathbb{R},
\end{array}
$$

where $\lambda\left(u-u_{x x}\right)(\lambda>0)$ is the dissipative term. They obtained the global existence result and blow-up results for strong solutions in Sobolev space $H^{s}$ with $s>3 / 2$ by Kato's theory. In [9], Lai and Wu also investigated the weakly dissipative Camassa-Holm equation:

$$
\begin{aligned}
u_{t}-u_{x x t} & +2 k u_{x}+3 u u_{x}+\lambda u^{2 n+1} \\
& -\beta u^{2 m} u_{x x}=2 u_{x} u_{x x}+u u_{x x x},
\end{aligned}
$$

where $k, \lambda, \beta \geq 0$ are constants, $n, m$ are natural numbers, and $\lambda u^{2 n+1}-\beta u^{2 m} u_{x x}$ is the weakly dissipative term. They obtained the local well-posedness in Sobolev space $H^{s}$ with $s>3 / 2$ by using the pseudoparabolic regularization technique and some estimates derived from the equation itself and also developed a sufficient condition which guaranteed the existence of weak solutions in Sobolev space $H^{s}$ with $1<s \leq 3 / 2$. We note that they only studied the equation in Sobolev space $H^{s}$.

On the other hand, the Camassa-Holm equation also admits many integrable multicomponent generalizations [20-32]. For example,

$$
\begin{gathered}
m_{t}+2 k u_{x}+u m_{x}+2 u_{x} m+\sigma \rho \rho_{x}=0 \\
\rho_{t}+(\rho u)_{x}=0
\end{gathered}
$$

where $m=u-u_{x x}$. The above system was derived in [33] with $\sigma=1$. In [20], Constantin and Ivanov gave a rigorous justification of the system which is a valid approximation to the governing equation for shallow water waves for $k=0$. The $u(x, t)$ represents the horizontal velocity of the fluid the $\rho(x, t)$ is related to the free surface elevation from equilibrium with boundary assumptions, and $u \rightarrow 0$ and $\rho \rightarrow 1$ as $|x| \rightarrow \infty$. They obtained the global well-posedness with small initial data and the conditions for wave-breaking mechanism to the system. For the case $\sigma=-1$, it is not physical as it corresponds to gravity pointing upwards, and $\rho \rightarrow 0$ as $x \rightarrow \infty$. The blow-up conditions are discussed in [25]. For $k=0$, Gui and Liu [27] established the local well-posedness for the system in a range of Besov spaces $B_{p, r}^{s} \times B_{p, r}^{s-1}$ with $s>\max (3 / 2,1+1 / p)$. They also derived wavebreaking mechanisms and the exact blow-up rate for strong solutions to the system in $H^{s} \times H^{s-1}$ with $s>3 / 2$. Tian et al. [34] obtained the local well-posedness for the system in Besov spaces $B_{p, r}^{s} \times B_{p, r}^{s}$ with $s>\max (3 / 2,1+1 / p)$. They also derived wave-breaking mechanisms for solutions and a result of blow-up solutions with certain profile in spaces $H^{s} \times H^{s}$ with $s>3 / 2$. Yan and Yin [21] investigated the 2component Degasperis-Procesi system which is similar to the 2-component Camassa-Holm system. They obtained the local well-posedness in Besov spaces and derived a precise blow-up scenario for strong solutions. Guan and Yin [23] presented a new global existence result and several new blow-up results of strong solutions to an integrable 2-component CamassaHolm shallow water system.

In fact, the 2-component Camassa-Holm system also admits some generalizations due to the energy dissipation mechanisms in a real world. In [35], Chen et al. investigated the weakly dissipative 2-component Camassa-Holm system:

$$
\begin{gathered}
u_{t}-u_{x x t}+2 k u_{x}+3 u u_{x}-\rho \rho_{x}+\lambda u^{2 n+1}-\beta u_{x x} \\
=2 u_{x} u_{x x}+u u_{x x x}, \quad t>0, x \in \mathbb{R}, \\
\rho_{t}+(\rho u)_{x}=0, \quad t>0, x \in \mathbb{R},
\end{gathered}
$$

where $k, \lambda, \beta \geq 0$ are constants and $n$ is a natural number. They investigated the local well-posedness for the system with initial data $\left(u_{0}, \rho_{0}\right) \in H^{s} \times H^{s-1}$ with $s \geq 2$ by Kato's theory and derived a precise blow-up scenario for strong solutions to the system.

Motivated by the work in $[9,21,23,27,33,35-38]$, we study the weakly dissipative Camassa-Holm system (1). We note that the Cauchy problem of system (1) in Besov spaces has not been discussed yet. We state our main tasks with three aspects. Firstly, we establish the local well-posedness of solutions to the system (1). Secondly, we present the precise blow-up criterions and exact blow-up rate for strong solutions. At last, we derive a global existence result of strong solutions. Because of the presence of high order nonlinear terms $u^{2 n+1}$ and $u^{2 m} u_{x x}$, the system (1) loses the conservation law $E=\int_{\mathbb{R}}\left(u^{2}+u_{x}^{2}\right) \mathrm{d} x$ which plays an important role in studying system (1).

Now we rewrite system (1) as

$$
\begin{gathered}
u_{t}+u u_{x}=P(D)\left[u^{2}+\frac{u_{x}^{2}}{2}+2 k u+\frac{1}{2} \rho^{2}\right] \\
+P_{1}(D)\left[-\lambda u^{2 n+1}+\beta u^{2 m} u_{x x}\right], \\
t>0, x \in \mathbb{R}, \\
\rho_{t}+(\rho u)_{x}=0, \quad t>0, x \in \mathbb{R}, \\
u(x, 0)=u_{0}(x), \quad x \in \mathbb{R}, \\
\rho(x, 0)=\rho_{0}(x), \quad x \in \mathbb{R},
\end{gathered}
$$

where the operators $P(D)=-\partial_{x}\left(1-\partial_{x x}\right)^{-1}$ and $P_{1}(D)=(1-$ $\left.\partial_{x x}\right)^{-1}$. We define the space:

$$
\begin{aligned}
& E_{p, r}^{s}(T) \\
& \quad= \begin{cases}C\left([0, T] ; B_{p, r}^{s}\right) \cap C^{1}\left([0, T] ; B_{p, r}^{s-1}\right), & 1 \leq r<\infty, \\
L^{\infty}\left([0, T] ; B_{p, \infty}^{s}\right) \cap \operatorname{lip}\left([0, T] ; B_{p, \infty}^{s-1}\right), & r=\infty,\end{cases}
\end{aligned}
$$

with $T>0, s \in \mathbb{R}, p \in[1, \infty]$, and $r \in[1, \infty]$.

The main results of this paper are stated as follows. Firstly, we present the local well-posedness theorem. 
Theorem 1. For $1 \leq p, r \leq \infty$ and $s>\max (3 / 2,1+1 / p)$. Let $\left(u_{0}, \rho_{0}-1\right) \in B_{p, r}^{s} \times B_{p, r}^{s-1}$. There exists a time $T>0$ such that the initial value problem (1) has a unique solution ( $u, \rho-$ 1) $\in E_{p, r}^{s}(T) \times E_{p, r}^{s-1}(T)$, and the map $\left(u_{0}, \rho_{0}-1\right) \rightarrow(u, \rho-$ $1)$ is continuous from a neighborhood of $\left(u_{0}, \rho_{0}-1\right)$ in $B_{p, r}^{s} \times$ $B_{p, r}^{s-1}$ into $C\left([0, T] ; B_{p, r}^{s^{\prime}}\right) \cap C^{1}\left([0, T] ; B_{p, r}^{s^{\prime}-1}\right) \times C\left([0, T] ; B_{p, r}^{s^{\prime}-1}\right) \cap$ $C^{1}\left([0, T] ; B_{p, r}^{s^{\prime}-2}\right)$ for every $s^{\prime}<s$ when $r=+\infty$ and $s^{\prime}=s$ whereas $r<+\infty$.

We obtain the following blow-up results.

Theorem 2. Let $\left(u_{0}, \rho_{0}-1\right) \in H^{s} \times H^{s-1}$ with $s>5 / 2$ and $T>0$ be the maximal existence time of the solution $(u, \rho)$ to system (1) with initial data $\left(u_{0}, \rho_{0}\right)$. Then, the corresponding solution blows up in finite time if and only if

$$
\lim _{t \rightarrow T^{-}} \inf _{x \in \mathbb{R}} u_{x}(t, x)=-\infty
$$

or

$\lim _{t \rightarrow T^{-}} \sup \left\|\rho_{x}(t, \cdot)\right\|_{L^{\infty}}=+\infty$.

Theorem 3. Let $m=0$ in system (1). Assume $\left(u_{0}, \rho_{0}-1\right) \epsilon$ $H^{s} \times H^{s-1}$ with $s>3 / 2$ and the initial value $\left(u_{0}, \rho_{0}\right)$ satisfies $u_{0 x}\left(x_{0}\right)<-\beta-\sqrt{2 K}$, where $K$ is a fixed constant defined in (95) and $\rho_{0}\left(x_{0}\right)=0$ with the point $x_{0}$ defined by $u_{0 x}\left(x_{0}\right)=$ $\inf _{x \in \mathbb{R}} u_{0 x}(x)$. Then, the corresponding solution $(u, \rho-1)$ to system (1) with $m=0$ blows up in finite time. Namely, there exists a $T_{0}$ with $0<T_{0} \leq-2 /(1-\delta)\left(u_{0 x}\left(x_{0}\right)+\beta\right)$ such that

$$
\lim \inf _{t \rightarrow T_{0}^{-}} \inf _{x \in \mathbb{R}} u_{x}(t, x)=-\infty,
$$

where $\delta \in(0,1)$ such that $-\sqrt{\delta} m_{1}(0)=\sqrt{2 K}$.

Theorem 4. Let $k=0$ in system (1). Assume $\left(u_{0}, \rho_{0}-1\right) \in$ $H^{s} \times H^{s-1}$ with $s>5 / 2$ and the initial value $\left(u_{0}, \rho_{0}\right)$ satisfies that $u_{0}$ is odd, $\rho_{0}$ is even, and $u_{0 x}(0)<-\sqrt{2 K_{1}}$, where $K_{1}$ is a fixed constant defined in (109) and $\rho_{0}(0)=0$. Then the corresponding solution $(u, \rho-1)$ to system (1) with $k=0$ blows up in finite time. More precisely, there exists a $T_{1}$ with $0<T_{1} \leq-2 /\left(1-\delta_{1}\right) u_{0 x}(0)$ such that

$$
\lim \inf _{t \rightarrow T_{1}^{-}} u_{x}(t, 0)=-\infty .
$$

In addition, if $\rho_{0 x}\left(x_{0}\right) \neq 0$ with some $x_{0} \in \mathbb{R}$ satisfying $u_{0 x}\left(x_{0}\right)=\inf _{x \in \mathbb{R}} u_{0 x}(x)$, then there exists a $T_{2}$ with $0<T_{2} \leq$ $-\left(2 /\left(\left(1-\delta_{1}\right) u_{0 x}(0)\right)\right)$ such that

(i) : $\quad \lim \sup _{\mathrm{t} \rightarrow \mathrm{T}_{2}^{-} \mathrm{x} \in \mathbb{R}} \rho_{\mathrm{x}}(\mathrm{t}, \mathrm{x})=+\infty \quad$ if $\rho_{0 \mathrm{x}}\left(\mathrm{x}_{0}\right)>0$,

(ii) : $\quad \lim \inf _{t \rightarrow T_{2}^{-}} \inf _{x \in \mathbb{R}} \rho_{x}(t, x)=-\infty$ if $\rho_{0 x}\left(x_{0}\right)<0$,

where $\delta_{1} \in(0,1)$ such that $-\sqrt{\delta_{1}} M(0)=\sqrt{2 K_{1}}$.

We also obtain the exact blow-up rate of strong solutions to system (1).
Theorem 5. Let $T<\infty$ be the maximal existence time of the corresponding solution $(u, \rho-1)$ to system (1) with $m=0$. The initial data $\left(u_{0}, \rho_{0}-1\right) \in H^{s} \times H^{s-1}$ with $s>3 / 2$ satisfies $u_{0 x}\left(x_{0}\right)<-\beta-\sqrt{2 K_{2}}$, where $K_{2}$ is a fixed constant defined in $(120)$ and $\rho_{0}\left(x_{0}\right)=0$ with the point $x_{0}$ defined by $u_{0}\left(x_{0}\right)=$ $\inf _{x \in \mathbb{R}} u_{0 x}(x)$; then

$$
\lim _{t \rightarrow T^{-}}\left[\inf _{x \in \mathbb{R}}\left(u_{x}(t, x)+\beta\right)(T-t)\right]=-2 .
$$

Now we present a global existence result of strong solutions to system (1).

Theorem 6. Let $m=0$ in system $(1)$ and $\left(u_{0}, \rho_{0}-1\right) \in H^{2} \times H^{1}$. If $\rho_{0}(x) \neq 0$ for all $x \in \mathbb{R}$, then the corresponding strong solution $(u, \rho-1)$ to system (1) with $m=0$ exists globally in time.

The remainder of this paper is organized as follows. In Section 2, some properties of Besov space and a priori estimates for solutions of transport equation are reviewed. Section 3 is devoted to the proof of Theorem 1 . The proofs of wave-breaking results and the precise blow-up rate of strong solutions to system (1) are given in Section 4, respectively. The proof of Theorem 6 is presented in Section 5.

Notation. In this paper, we denote the convolution on $\mathbb{R}$, by $*$ the norm of Lebesgue space $L^{p}$ by $\|\cdot\|_{L^{p}}, 1 \leq p \leq \infty$ and the norm in Sobolev space $H^{s}, s \in \mathbb{R}$, by $\|\cdot\|_{H^{s}}$, and the norm in Besov space $B_{p, r}^{s}, s \in \mathbb{R}$, by $\|\cdot\|_{B_{p, r}^{s}}$. Here we denote $a+=a+\varepsilon$, where $\varepsilon>0$ is a sufficiently small number.

\section{Preliminary}

In this section, we recall some basic facts in Besov space. One may check [21, 39-42] for more details.

Proposition 7 (see $[40,42]$ ). Let $s \in \mathbb{R}, 1 \leq p$, and $r \leq+\infty$. The nonhomogeneous Besov space is defined by $B_{p, r}^{s}=\{f \in$ $\left.S^{\prime}\left(\mathbb{R}^{n}\right) \mid\|f\|_{B_{p, r}^{s}}<+\infty\right\}$, where

$$
\|f\|_{B_{p, r}^{s}}= \begin{cases}\left(\sum_{j=-1}^{\infty} 2^{j r s}\left\|\Delta_{j} f\right\|_{L^{p}}^{r}\right)^{1 / r}, & r<\infty, \\ \sup _{j \geq-1}^{j s}\left\|\Delta_{j} f\right\|_{L^{p}}, & r=\infty .\end{cases}
$$

Moreover, $S_{j} f=\sum_{q=-1}^{j-1} \Delta_{q} f$.

Proposition 8 (see $[40,42]$ ). Let $s \in \mathbb{R}, 1 \leq p, r, p_{j}, r_{j} \leq \infty$, and $j=1,2$, then; consider the following.

(1) Embedding: $B_{p_{1}, r_{1}}^{s} \hookrightarrow B_{p_{2}, r_{2}}^{s-n\left(1 / p_{1}-1 / p_{2}\right)}$, if $p_{1} \leq p_{2}$ and $r_{1} \leq r_{2}$. And $B_{p, r_{2}}^{s_{2}} \hookrightarrow B_{p, r_{1}}^{s_{1}}$ locally compact if $s_{1} \leq s_{2}$.

(2) Algebraic properties: for any $s>0, B_{p, r}^{s} \cap L^{\infty}$ is an algebra. $B_{p, r}^{s}$ is an algebra $\Leftrightarrow B_{p, r}^{s} \hookrightarrow L^{\infty} \Leftrightarrow s>$ $n / p$ or $s \geq n / p$ and $r=1$. 
(3) Complex interpolation:

$$
\|f\|_{B_{p, r}^{\theta s_{1}+(1-\theta) s_{2}}} \leq C\|f\|_{B_{p, r}^{s_{1}}}^{\theta}\|f\|_{B_{p, r}^{s_{2}}}^{1-\theta},
$$

$$
\text { for every } f \in B_{p, r}^{s_{1}} \cap B_{p, r}^{s_{2}}, \theta \in[0,1] \text {. }
$$

(4) Fatou's Lemma: if $\left(u_{n}\right)_{n \in \mathbb{N}}$ is bounded in $B_{p, r}^{s}$ and $u_{n} \rightarrow$ $u$ in $S^{\prime}(\mathbb{R})$, then $u \in B_{p, r}^{s}$ and

$$
\|u\|_{B_{p, r}^{s}} \leq \lim _{n \rightarrow \infty} \inf \left\|u_{n}\right\|_{B_{p, r}^{s}}
$$

(5) Let $m \in \mathbb{R}$ and $f$ be an $S^{m}$-multiplier (i.e., $f: \mathbb{R}^{n} \rightarrow$ $\mathbb{R}$ is smooth and satisfies that for every $\alpha \in \mathbb{N}^{n}$ there exists a constant $C_{\alpha}$ such that $\left|\partial^{\alpha} f(\xi)\right| \leq C_{\alpha}(1+$ $|\xi|)^{m-|\alpha|}$ for all $\left.\xi \in \mathbb{R}^{n}\right)$. Then, the operator $f(D)$ is continuous from $B_{p, r}^{s}$ to $B_{p, r}^{s-m}$.

(6) Density: $C_{c}^{\infty}$ is dense in $B_{p, r}^{s} \Leftrightarrow 1 \leq p, r<\infty$.

Lemma 9 (see [40]). Let I be an open interval of $\mathbb{R}$. Let $s>0$ and $s_{1}$ be the smallest integer such that $s_{1} \geq s$. Let $F: I \rightarrow \mathbb{R}$ satisfy $F(0)=0$ and $F^{\prime} \in W^{s_{1}, \infty}(I ; \mathbb{R})$. Assume that $v \in B_{p, r}^{s}$ has values in $J \subset \subset I$. Then, $F(v) \in B_{p, r}^{s}$ and there exists a constant $C$ depending only on $s, I, J, N$ such that

$$
\|F(v)\|_{B_{p, r}^{s}} \leq C\left(1+\|v\|_{L^{\infty}}\right)^{s_{1}}\left\|F^{\prime}\right\|_{W^{s_{1}, \infty}(I)}\|v\|_{B_{p, r}^{s}} .
$$

Lemma 10 (see [40]). Let I be an open interval of $\mathbb{R}$. Let $s>0$ and $s_{1}$ be the smallest integer such that $s_{1} \geq s$. Let $F: I \rightarrow \mathbb{R}$ satisfy $F^{\prime}(0)=0$ and $F^{\prime \prime} \in W^{s_{1}, \infty}(I ; \mathbb{R})$. Assume that $u, v \in B_{p, r}^{s}$ has values in $J \subset \subset I$. Then, there exists a constant $C$ depending only on $s, I, J, N$ such that

$$
\begin{aligned}
& \|F(u)-F(v)\|_{B_{p, r}^{s}} \\
& \leq C\left(1+\|u\|_{L^{\infty}}\right)^{s_{1}}\left\|F^{\prime \prime}\right\|_{W^{s_{1}, \infty}(I)} \\
& \quad \times\left(\|u-v\|_{B_{p, r}^{s}} \sup _{\theta \in[0,1]}\|\theta u+(1-\theta) v\|_{L^{\infty}}\right. \\
& \left.\quad+\|u-v\|_{L^{\infty}} \sup _{\theta \in[0,1]}\|\theta u+(1-\theta) v\|_{B_{p, r}^{s}}\right) .
\end{aligned}
$$

Lemma 11 (see [40]). Assume that $1 \leq p$ and $r \leq \infty$; the following estimates hold:

(i) for $s>0$, then

$$
\|f g\|_{B_{p, r}^{s}} \leq C\left(\|f\|_{B_{p, r}^{s}}\|g\|_{L^{\infty}}+\|f\|_{L^{\infty}}\|g\|_{B_{p, r}^{s}}\right) ;
$$

(ii) for $s_{1} \leq 1 / p, s_{2}>1 / p\left(s_{2} \geq(1 / p)\right.$ if $\left.r=1\right)$, and $s_{1}+s_{2}>0$, then

$$
\|f g\|_{B_{p, r}^{s_{1}}} \leq C\|f\|_{B_{p, r}^{s_{1}}}\|g\|_{B_{p, r}^{s},}
$$

where $C$ are constants independent of $f, g$.
Then we present two related lemmas for the following transport equations:

$$
\begin{gathered}
f_{t}+d \cdot \nabla f=F, \\
\left.f\right|_{t=0}=f_{0},
\end{gathered}
$$

where $d: \mathbb{R} \times \mathbb{R}^{n} \rightarrow \mathbb{R}^{n}$ stands for a given time dependent vector field and $f_{0}: \mathbb{R}^{n} \rightarrow \mathbb{R}^{m}$ and $F: \mathbb{R} \times \mathbb{R}^{n} \rightarrow \mathbb{R}^{m}$ are known data.

Lemma 12 (see $[39,40])$. Let $1 \leq p \leq p_{1} \leq \infty, 1 \leq r \leq \infty$, and $p^{\prime}=p /(p-1)$. Assume that $s>-n \cdot \min \left(1 / p_{1}, 1 / p^{\prime}\right)$ or $s>-1-n \cdot \min \left(1 / p_{1}, 1 / p^{\prime}\right)$ if $\nabla \cdot d=0$. Then, there exists a constant $C$ depending only on $n, p, p_{1}, r, s$, such that the following estimate holds true:

$$
\begin{aligned}
& \|f\|_{\tilde{L}_{t}^{\infty}\left([0, t] ; B_{p, r}^{s}\right)} \\
& \leq e^{C_{1} \int_{0}^{t} Z(\tau) \mathrm{d} \tau} \\
& \quad \times\left[\left\|f_{0}\right\|_{B_{p, r}^{s}}+\int_{0}^{t} e^{-C_{1} \int_{0}^{\tau} Z(\xi) d \xi}\|F(\tau)\|_{B_{p, r}^{s}} \mathrm{~d} \tau\right],
\end{aligned}
$$

with

$$
\begin{aligned}
& Z(t) \\
& \quad= \begin{cases}\|\nabla d(t)\|_{B_{p_{1}, \infty}^{n / p_{1} \cap L^{\infty}}}, & s<1+\frac{n}{p_{1}}, \\
\|\nabla d(t)\|_{B_{p_{1}, r}^{s-1},}, & s>1+\frac{n}{p_{1}} \text { or } s=1+\frac{n}{p_{1}}, r=1 .\end{cases}
\end{aligned}
$$

If $f=d$, then for all $s>0(\nabla \cdot d=0, s>-1),(22)$ holds with $Z(t)=\|\nabla d(t)\|_{L^{\infty}}$.

Let us state the existence result for transport equation with data in Besov space.

Lemma 13 (see [40]). Let $p, p_{1}, r, s$ be as in the statement of Lemma 12 and $f_{0} \in B_{p, r}^{s}$ and $F \in L^{1}\left([0, T] ; B_{p, r}^{s}\right) ; d \in$ $L^{\rho}\left([0, T] ; B_{\infty, \infty}^{-M}\right)$ is a time dependent vector field for some $\rho>1, M>0$, such that if $s<1+n / p_{1}$ then $\nabla d \epsilon$ $L^{1}\left([0, T] ; B_{p_{1}, \infty}^{n / p_{1}} \cap L^{\infty}\right)$; if $s>1+n / p_{1}$ or $s=1+n / p_{1}, r=1$ then $\nabla d \in L^{1}\left([0, T] ; B_{p_{1}, r}^{s-1}\right)$. Thus transport equation (21) has a unique solution $f \in L^{\infty}\left([0, T] ; B_{p, r}^{s}\right) \bigcap\left(\bigcap_{s^{\prime}<s} C\left([0, T] ; B_{p, 1}^{s^{\prime}}\right)\right)$, and (22) holds true. If $r<\infty$, then one has $f \in C\left([0, T] ; B_{p, r}^{s}\right)$.

\section{The Proof of Theorem 1}

We finish the proof of Theorem 1 by the following steps. 
3.1. Existence of Solutions. For convenience, we denote $\eta=$ $\rho-1$ and rewrite (7) as

$$
\begin{array}{r}
u_{t}+u u_{x}=P(D)\left[u^{2}+\frac{u_{x}^{2}}{2}+2 k u+\frac{\eta^{2}}{2}+\eta\right] \\
+P_{1}(D)\left[-\lambda u^{2 n+1}+\beta u^{2 m} u_{x x}\right], \\
t>0, x \in \mathbb{R}, \\
\eta_{t}+u \eta_{x}=-u_{x} \eta-u_{x}, \quad t>0, x \in \mathbb{R}, \\
u(x, 0)=u_{0}(x), \quad x \in \mathbb{R}, \\
\eta(x, 0)=\eta_{0}(x)=\rho_{0}(x)-1, \quad x \in \mathbb{R} .
\end{array}
$$

We use a standard iterative process to construct the approximate solutions to (24)

Step 1. Starting from $u^{0}=\eta^{0}=0$, we define by induction a sequence of smooth functions $\left(u^{i}, \eta^{i}\right)_{i \in \mathbb{N}} \in C\left(\mathbb{R}^{+} ; B_{p, r}^{\infty}\right)^{2}$ solving the following transport equation:

$$
\begin{gathered}
\left(\partial_{t}+u^{i} \partial_{x}\right) u^{i+1}=F(t, x), \quad t>0, \quad x \in \mathbb{R}, \\
\left(\partial_{t}+u^{i} \partial_{x}\right) \eta^{i+1}=-\eta^{i} \partial_{x} u^{i}-\partial_{x} u^{i}, \quad t>0, x \in \mathbb{R}, \\
u^{i+1}(x, 0)=u_{0}^{i+1}(x)=S_{i+1} u_{0}, \quad x \in \mathbb{R}, \\
\eta^{i+1}(x, 0)=\eta_{0}^{i+1}(x)=S_{i+1} \eta_{0}, \quad x \in \mathbb{R},
\end{gathered}
$$

where

$$
\begin{aligned}
F(t, x)= & P(D)\left[\left(u^{i}\right)^{2}+\frac{1}{2}\left(u^{i}\right)_{x}^{2}+2 k u^{i}+\frac{1}{2}\left(\eta^{i}\right)^{2}+\eta^{i}\right] \\
& +P_{1}(D)\left[-\lambda\left(u^{i}\right)^{2 n+1}+\beta\left(u^{i}\right)^{2 m}\left(u^{i}\right)_{x x}\right] .
\end{aligned}
$$

Since all the data $S_{i+1} u_{0}, S_{i+1} \eta_{0} \in B_{p, r}^{\infty}$, Lemma 13 enables us to show that for all $i \in \mathbb{N}$, the system (25) has a global solution which belongs to $C\left(\mathbb{R}^{+} ; B_{p, r}^{\infty}\right)^{2}$.

Step 2. Next, we prove that $\left(u^{i}, \eta^{i}\right)_{i \in \mathbb{N}}$ is uniformly bounded in $E_{p, r}^{\mathcal{s}}(T) \times E_{p, r}^{\mathcal{s}-1}(T)$.
According to Lemma 12, we have the following inequalities for all $i \in \mathbb{N}$ :

$$
\begin{aligned}
& \left\|u^{i+1}\right\|_{B_{p, r}^{s}} \\
& \leq e^{C_{1} \int_{0}^{t}\left\|u^{i}(\tau)\right\|_{B_{p, r}^{s}} \mathrm{~d} \tau} \\
& \times\left[\left\|u_{0}\right\|_{B_{p, r}^{s}}+\int_{0}^{t} e^{-C_{1} \int_{0}^{\tau}\left\|u^{i}(\xi)\right\|_{B_{p, r}^{s}} \mathrm{~d} \xi}\|F(\tau, \cdot)\|_{B_{p, r}^{s}} \mathrm{~d} \tau\right], \\
& \left\|\eta^{i+1}\right\|_{B_{p, r}^{s-1}} \leq e^{C_{2} \int_{0}^{t}\left\|u^{i}\right\|_{B_{p, r}^{s}}^{s} \mathrm{~d} \tau}\left[\left\|\eta_{0}\right\|_{B_{p, r}^{s-1}}+\int_{0}^{t} e^{-C_{2} \int_{0}^{\tau}\left\|u^{i}\right\|_{B_{p, r}^{s}}^{s} \mathrm{~d} \xi}\right. \\
& \left.\times\left\|-\eta^{i} \partial_{x} u^{i}-\partial_{x} u^{i}\right\|_{B_{p, r}^{s-1}} \mathrm{~d} \tau\right] .
\end{aligned}
$$

From Proposition 8, Lemma 11, and $S^{-1}$-multiplier property of $P(D)$, it yields that

$$
\begin{aligned}
\| P(D) & {\left[\left(u^{i}\right)^{2}+\frac{1}{2}\left(u^{i}\right)_{x}^{2}+2 k u^{i}+\frac{1}{2}\left(\eta^{i}\right)^{2}+\eta^{i}\right] \|_{B_{p, r}^{s}} } \\
\leq & C\left(\left\|u^{i}\right\|_{B_{p, r}^{s}}^{2}+2 k\left\|u^{i}\right\|_{B_{p, r}^{s}}+\left\|\eta^{i}\right\|_{B_{p, r}^{s-1}}^{2}+\left\|\eta^{i}\right\|_{B_{p, r}^{s-1}}\right) .
\end{aligned}
$$

Using the $S^{-2}$-multiplier property of $P_{1}(D)$, we get

$$
\begin{gathered}
\left\|P_{1}(D)\left[-\lambda\left(u^{i}\right)^{2 n+1}+\beta\left(u^{i}\right)^{2 m} u_{x x}^{i}\right]\right\|_{B_{p, r}^{s}} \\
\leq C\left\|-\lambda\left(u^{i}\right)^{2 n+1}+\beta\left(u^{i}\right)^{2 m} u_{x x}^{i}\right\|_{B_{p, r}^{s-2}} .
\end{gathered}
$$

(i) By the assumption of $f\left(u^{i}\right)=\left(u^{i}\right)^{2 n+1}$ and Lemma 9, we have

$$
\begin{aligned}
& \left\|-\lambda\left(u^{i}\right)^{2 n+1}\right\|_{B_{p, r}^{s-2}} \\
& \leq C\left(1+\left\|u^{i}\right\|_{L^{\infty}}\right)^{(s-1)_{1}}\left\|f^{\prime}\right\|_{W^{(s-1)_{1}, \infty}}\left\|u^{i}\right\|_{B_{p, r}^{s-1}} \\
& \leq C\left(1+\left\|u^{i}\right\|_{B_{p, r}^{s}}\right)^{(s-1)_{1}}\left\|u^{i}\right\|_{B_{p, r}^{s} \cdot} .
\end{aligned}
$$

(ii) As we know, if $\max (3 / 2,1+1 / p)<s \leq 2+1 / p$, then $B_{p, r}^{s-1}$ is an algebra; if $s>2+1 / p$, then $B_{p, r}^{s-2}$ is an algebra. By the assumption of $h\left(u^{i}\right)=\left(u^{i}\right)^{2 m}$ and Lemmas 10 and 11, we deduce that

$$
\begin{aligned}
\left\|\beta\left(u^{i}\right)^{2 m} u_{x x}^{i}\right\|_{B_{p, r}^{s-2}} \leq & C \beta\left\|\left(u^{i}\right)^{2 m}\right\|_{B_{p, r}^{s-1}}\left\|u_{x x}^{i}\right\|_{B_{p, r}^{s-2}} \\
\leq & C\left(1+\left\|u^{i}\right\|_{B_{p, r}^{s-1}}\right)^{(s-1)_{1}} \\
& \times\left\|u^{i}\right\|_{B_{p, r}^{s-1}}\left\|u_{x x}^{i}\right\|_{B_{p, r}^{s-2}} \\
\leq & C\left(1+\left\|u^{i}\right\|_{B_{p, r}^{s}}\right)^{s_{1}}\left\|u^{i}\right\|_{B_{p, r}^{s}} .
\end{aligned}
$$


Combining (i) and (ii) yields

$$
\begin{gathered}
\left\|P_{1}(D)\left[-\lambda\left(u^{i}\right)^{2 n+1}+\beta\left(u^{i}\right)^{2 m}\left(u^{i}\right)_{x x}\right]\right\|_{B_{p, r}^{s}} \\
\leq C\left(1+\left\|u^{i}\right\|_{B_{p, r}^{s}}\right)^{s_{1}}\left\|u^{i}\right\|_{B_{p, r}^{s}} .
\end{gathered}
$$

Thanks to Lemma 11, we get

$$
\left\|-\eta^{i} \partial_{x} u^{i}-\partial_{x} u^{i}\right\|_{B_{p, r}^{s-1}} \leq\left\|u^{i}\right\|_{B_{p, r}^{s}}\left\|\eta^{i}\right\|_{B_{p, r}^{s-1}}+\left\|u^{i}\right\|_{B_{p, r}^{s}} .
$$

Therefore from (27) to (34), we obtain

$$
\begin{aligned}
& \left\|u^{i+1}\right\|_{B_{p, r}^{s}}+\left\|\eta^{i+1}\right\|_{B_{p, r}^{s-1}} \\
& \leq C_{3} \cdot e^{C_{3} \int_{0}^{t}\left\|u^{i}\right\|_{B_{p, r}^{s}}^{s} \mathrm{~d} \tau} \\
& \times\left[\left\|u_{0}\right\|_{B_{p, r}^{s}}+\left\|\eta_{0}\right\|_{B_{p, r}^{s-1}}\right. \\
& \quad+\int_{0}^{t} e^{-C_{3} \int_{0}^{\tau}\left\|u^{i}\right\|_{B_{p, r}^{s}}^{s} \mathrm{~d} \xi}\left(\left\|u^{i}\right\|_{B_{p, r}^{s}}+\left\|\eta^{i}\right\|_{B_{p, r}^{s-1}}+k+1\right)^{s_{1}} \\
& \left.\quad \times\left(\left\|u^{i}\right\|_{B_{p, r}^{s}}+\left\|\eta^{i}\right\|_{B_{p, r}^{s-1}}\right)\right] \mathrm{d} \tau .
\end{aligned}
$$

Let us choose a $T>0$ such that $2 s_{1} C_{3}^{s_{1}+1}\left(\left\|u_{0}^{i}\right\|_{B_{p, r}^{s}}+\left\|\eta_{0}^{i}\right\|_{B_{p, r}^{s-1}}+\right.$ $k+1)^{s_{1}} T<1$ and

$$
\begin{aligned}
(k+1 & \left.+\left\|u^{i}\right\|_{B_{p, r}^{s}}+\left\|\eta^{i}\right\|_{B_{p, r}^{s-1}}\right)^{s_{1}} \\
& \leq \frac{C_{3}^{s_{1}}\left(k+1+\left\|u_{0}\right\|_{B_{p, r}^{s}}+\left\|\eta_{0}\right\|_{B_{p, r}^{s-1}}\right)^{s_{1}}}{1-2 s_{1} C_{3}^{s_{1}+1}\left(k+1+\left\|u_{0}\right\|_{B_{p, r}^{s}}+\left\|\eta_{0}\right\|_{B_{p, r}^{s-1}}\right)^{s_{1}} t} .
\end{aligned}
$$

Inserting (36) into (35) yields

$$
\begin{aligned}
(k+1 & \left.+\left\|u^{i+1}\right\|_{B_{p, r}^{s}}+\left\|\eta^{i+1}\right\|_{B_{p, r}^{s-1}}\right)^{s_{1}} \\
& \leq \frac{C_{3}^{s_{1}}\left(k+1+\left\|u_{0}\right\|_{B_{p, r}^{s}}+\left\|\eta_{0}\right\|_{B_{p, r}^{s-1}}\right)^{s_{1}}}{1-2 s_{1} C_{3}^{s_{1}+1}\left(k+1+\left\|u_{0}\right\|_{B_{p, r}^{s}}+\left\|\eta_{0}\right\|_{B_{p, r}^{s-1}}\right)^{s_{1}} t} .
\end{aligned}
$$

Therefore, $\left(u^{i}, \eta^{i}\right)_{i \in \mathbb{N}}$ is uniformly bounded in $C\left([0, T] ; B_{p, r}^{s}\right) \times$ $C\left([0, T] ; B_{p, r}^{s-1}\right)$. From Proposition 8, Lemma 11, and the following embedding properties:

$$
B_{p, r}^{s} \hookrightarrow B_{p, r}^{s-1}, \quad B_{p, r}^{s-1} \hookrightarrow B_{p, r}^{s-2},
$$

we have

$$
\begin{aligned}
\left\|u^{i} \partial_{x} u^{i+1}\right\|_{B_{p, r}^{s-1}} & \leq\left\|u^{i}\right\|_{B_{p, r}^{s-1}}\left\|\partial_{x} u^{i+1}\right\|_{B_{p, r}^{s-1}} \\
& \leq\left\|u^{i}\right\|_{B_{p, r}^{s}}\left\|u^{i+1}\right\|_{B_{p, r}^{s}}, \\
\left\|u^{i} \partial_{x} \eta^{i+1}\right\|_{B_{p, r}^{s-2}} & \leq\left\|u^{i}\right\|_{B_{p, r}^{s-1}}\left\|\partial_{x} \eta^{i+1}\right\|_{B_{p, r}^{s-2}} \\
& \leq\left\|u^{i}\right\|_{B_{p, r}^{s}}\left\|\eta^{i+1}\right\|_{B_{p, r}^{s-1}} .
\end{aligned}
$$

We deduce that $u^{i} \partial_{x} u^{i+1}$ and $F(t, x)$ are uniformly bounded in $C\left([0, T] ; B_{p, r}^{s-1}\right)$, in the same way that $u^{i} \partial_{x} \eta^{i+1},-\eta^{i} \partial_{x} u^{i}-$ $\partial_{x} u^{i}$ are uniformly bounded in $C\left([0, T] ; B_{p, r}^{s-2}\right)$. Using the system (25), we have $\left(\partial_{t} u^{i+1}, \partial_{t} \eta^{i+1}\right) \in C\left([0, T] ; B_{p, r}^{s-1}\right) \times$ $C\left([0, T] ; B_{p, r}^{s-2}\right)$ is uniformly bounded, which derives that $\left(u^{i}, \eta^{i}\right)_{i \in \mathbb{N}}$ is uniformly bounded in $E_{p, r}^{s}(T) \times E_{p, r}^{s-1}(T)$.

Step 3. Now we demonstrate that $\left(u^{i}, \eta^{i}\right)_{i \in \mathbb{N}}$ is a Cauchy sequence in $C\left([0, T] ; B_{p, r}^{s-1}\right) \times C\left([0, T] ; B_{p, r}^{s-2}\right)$.

In fact, according to (25), we note that for all $i, j \in \mathbb{N}$, we have

$$
\begin{aligned}
& \left(\partial_{t}+u^{i+j} \partial_{x}\right)\left(u^{i+j+1}-u^{i+1}\right) \\
& =\left(u^{i}-u^{i+j}\right) \partial_{x} u^{i+1}+P(D) \\
& \times\left[\left(u^{i+j}-u^{i}\right)\left(u^{i+j}+u^{i}\right)+\frac{1}{2} \partial_{x}\left(u^{i+j}-u^{i}\right)\right. \\
& \cdot \partial_{x}\left(u^{i+j}+u^{i}\right)+2 k\left(u^{i+j}-u^{i}\right)+\frac{1}{2}\left(\eta^{i+j}-\eta^{i}\right) \\
& \left.\times\left(\eta^{i+j}+\eta^{i}\right)+\left(\eta^{i+j}-\eta^{i}\right)\right] \\
& +P_{1}(D)\left[-\lambda\left(\left(u^{i+j}\right)^{2 n+1}-\left(u^{i}\right)^{2 n+1}\right)\right. \\
& \left.+\beta\left(\left(u^{i+j}\right)^{2 m}\left(u^{i+j}\right)_{x x}-\left(u^{i}\right)^{2 m}\left(u^{i}\right)_{x x}\right)\right] \\
& \left(\partial_{t}+u^{i+j} \partial_{x}\right)\left(\eta^{i+j+1}-\eta^{i+1}\right) \\
& =\left(u^{i}-u^{i+j}\right) \partial_{x} \eta^{i+1}-\left(\eta^{i+j}-\eta^{i}\right) \partial_{x} u^{i} \\
& -\eta^{i+j} \partial_{x}\left(u^{i+j}-u^{i}\right)-\partial_{x}\left(u^{i+j}-u^{i}\right) .
\end{aligned}
$$

(1) We estimate the terms in the right side of (40). By Lemma 11, we have

$$
\begin{gathered}
\left\|\left(u^{i}-u^{i+j}\right) \partial_{x} u^{i+1}\right\|_{B_{p, r}^{s-1}} \leq C\left\|u^{i+j}-u^{i}\right\|_{B_{p, r}^{s-1}}\left\|\partial_{x} u^{i+1}\right\|_{B_{p, r}^{s-1}}, \\
\left\|P(D)\left(u^{i+j}-u^{i}\right)\left(u^{i+j}+u^{i}\right)\right\|_{B_{p, r}^{s-1}} \\
\leq C\left\|u^{i+j}-u^{i}\right\|_{B_{p, r}^{s-1}}\left\|u^{i+j}+u^{i}\right\|_{B_{p, r}^{s-1}}, \\
\left\|P(D)\left[\partial_{x}\left(u^{i+j}-u^{i}\right) \partial_{x}\left(u^{i+j}+u^{i}\right)\right]\right\|_{B_{p, r}^{s-1}} \\
\leq C\left\|\partial_{x}\left(u^{i+j}-u^{i}\right)\right\|_{B_{p, r}^{s-2}}\left\|\partial_{x}\left(u^{i+j}+u^{i}\right)\right\|_{B_{p, r}^{s-1}}, \\
\left\|P(D)\left[2 k\left(u^{i+j}-u^{i}\right)\right]\right\|_{B_{p, r}^{s-1}} \leq C k\left\|u^{i+j}-u^{i}\right\|_{B_{p, r}^{s-2}}, \\
\left\|P(D)\left[\left(\eta^{i+j}-\eta^{i}\right)\left(\eta^{i+j}+\eta^{i}\right)\right]\right\|_{B_{p, r}^{s-1}} \\
\leq C\left\|\eta^{i+j}-\eta^{i}\right\|_{B_{p, r}^{s-2}}\left\|\eta^{i+j}+\eta^{i}\right\|_{B_{p, r}^{s-1}} \\
\left\|P(D)\left(\eta^{i+j}-\eta^{i}\right)\right\|_{B_{p, r}^{s-1}} \leq C\left\|\eta^{i+j}-\eta^{i}\right\|_{B_{p, r}^{s-2}} .
\end{gathered}
$$


We use Lemma 10 to estimate the high order terms in (40). Firstly, we get

$$
\begin{aligned}
& \left\|P_{1}(D)\left[-\lambda\left(\left(u^{i+j}\right)^{2 n+1}-\left(u^{i}\right)^{2 n+1}\right)\right]\right\|_{B_{p, r}^{s-1}} \\
& \leq C\left(1+\left\|u^{i+j}\right\|_{B_{p, r}^{s-1}}+\left\|u^{i}\right\|_{B_{p, r}^{s-1}}\right)^{s_{1}}\left\|u^{i+j}-u^{i}\right\|_{B_{p, r}^{s-1}} .
\end{aligned}
$$

Secondly, by $u^{2 m} u_{x x}=\partial_{x}\left(u^{2 m} u_{x}\right)-2 m u^{2 m-1} u_{x}^{2}$ and Lemma 11, we deduce that

$$
\begin{aligned}
& \left\|\beta\left(\left(u^{i+j}\right)^{2 m} u_{x x}^{i+j}-\left(u^{i}\right)^{2 m} u_{x x}^{i}\right)\right\|_{B_{p, r}^{s-3}} \\
& \leq C\left(\left\|\partial_{x}\left(\left(u^{i+j}\right)^{2 m} u_{x}^{i+j}-\left(u^{i}\right)^{2 m} u_{x}^{i}\right)\right\|_{B_{p, r}^{s-3}}\right. \\
& \left.\quad+\left\|\left(u^{i+j}\right)^{2 m-1}\left(u_{x}^{i+j}\right)^{2}-\left(u^{i}\right)^{2 m-1}\left(u_{x}^{i}\right)^{2}\right\|_{B_{p, r}^{s-3}}\right) .
\end{aligned}
$$

Hence,

$$
\begin{aligned}
& \left\|P_{1}(D) \beta\left[\left(u^{i+j}\right)^{2 m} u_{x x}^{i+j}-\left(u^{i}\right)^{2 m} u_{x x}^{i}\right]\right\|_{B_{p, r}^{s-1}} \\
& \leq C\left(1+\left\|u^{i+j}\right\|_{B_{p, r}^{s}}+\left\|u^{i}\right\|_{B_{p, r}^{s}}\right)^{s_{1}+1}\left\|u^{i+j}-u^{i}\right\|_{B_{p, r}^{s-1}} .
\end{aligned}
$$

(2) Now we estimate the right side of (41):

$$
\begin{gathered}
\left\|\left(u^{i}-u^{i+j}\right) \partial_{x} \eta^{i+1}\right\|_{B_{p, r}^{s-2}} \leq C\left\|u^{i+j}-u^{i}\right\|_{B_{p, r}^{s-1}}\left\|\partial_{x} \eta^{i+1}\right\|_{B_{p, r}^{s-2}}, \\
\left\|\left(\eta^{i+j}-\eta^{i}\right) \partial_{x} u^{i}\right\|_{B_{p, r}^{s-2}} \leq C\left\|\eta^{i+j}-\eta^{i}\right\|_{B_{p, r}^{s-2}}\left\|\partial_{x} u^{i}\right\|_{B_{p, r}^{s-1}}, \\
\left\|\eta^{i+j} \partial_{x}\left(u^{i+j}-u^{i}\right)\right\|_{B_{p, r}^{s-2}} \\
\leq C\left\|\partial_{x}\left(u^{i+j}-u^{i}\right)\right\|_{B_{p, r}^{s-2}}\left\|\eta^{i+j}\right\|_{B_{p, r}^{s-1}} \\
\left\|\partial_{x}\left(u^{i+j}-u^{i}\right)\right\|_{B_{p, r}^{s-2}} \leq C\left\|u^{i+j}-u^{i}\right\|_{B_{p, r}^{s-1}} .
\end{gathered}
$$

Combining (1), (2), and Lemma 12 yields for all $t \in[0, T]$

$$
\begin{aligned}
& \left\|u^{i+j+1}-u^{i+1}\right\|_{B_{p, r}^{s-1}} \\
& \leq e^{C \int_{0}^{t}\left\|u^{i+j}\right\|_{B_{p, r}^{s}} \mathrm{~d} \tau} \\
& \times\left\{\left\|u_{0}^{i+j+1}-u_{0}^{i+1}\right\|_{B_{p, r}^{s-1}}+C\right. \\
& \quad \times \int_{0}^{t} e^{-C \int_{0}^{\tau}\left\|u^{i+j}\right\|_{B_{p, r}^{s}} \mathrm{~d} \xi} \\
& \quad \times\left[\left(\left\|\eta^{i+j}-\eta^{i}\right\|_{B_{p, r}^{s-2}}\right)\left(\left\|\eta^{i+j}\right\|_{B_{p, r}^{s-1}}+\left\|\eta^{i}\right\|_{B_{p, r}^{s-1}}+1\right)\right. \\
& \quad+\left\|u^{i+j}-u^{i}\right\|_{B_{p, r}^{s-1}} \\
& \left.\left.\quad \times\left(\left\|u^{i}\right\|_{B_{p, r}^{s}}+\left\|u^{i+j}\right\|_{B_{p, r}^{s}}+\left\|u^{i+1}\right\|_{B_{p, r}^{s}}+k+1\right)^{s_{1}+1}\right] \mathrm{~d} \tau\right\},
\end{aligned}
$$

$\left\|\eta^{i+j+1}-\eta^{i+1}\right\|_{B_{p, r}^{s-2}}$

$$
\begin{aligned}
& \leq e^{C \int_{0}^{t}\left\|u^{i+j}\right\|_{B_{p, r}^{s}} \mathrm{~d} \tau} \\
& \times\left\{\left\|\eta_{0}^{i+j+1}-\eta_{0}^{i+1}\right\|_{B_{p, r}^{s-2}}\right. \\
& +C \int_{0}^{t} e^{-C \int_{0}^{\tau}\left\|u^{i+j}\right\|_{B_{p, r}^{s}} \mathrm{~d} \xi} \\
& \times\left[\left\|u^{i+j}-u^{i}\right\|_{B_{p, r}^{s-1}}\left(\left\|\eta^{i+1}\right\|_{B_{p, r}^{s-1}}+\left\|\eta^{i+j}\right\|_{B_{p, r}^{s-1}}+1\right)\right. \\
& \left.\left.\quad+\left(\left\|\eta^{i+j}-\eta^{i}\right\|_{B_{p, r}^{s-2}}\right)\left\|u^{i}\right\|_{B_{p, r}^{s}}\right] \mathrm{~d} \tau\right\} .
\end{aligned}
$$

Since $\left(u^{i}, \eta^{i}\right)_{i \in \mathbb{N}}$ is uniformly bounded in $E_{p, r}^{\mathcal{s}}(T) \times E_{p, r}^{s-1}(T)$ and

$$
\begin{aligned}
& u_{0}^{i+j+1}-u_{0}^{i+1}=\sum_{q=i+1}^{i+j} \Delta_{q} u_{0} \\
& \eta_{0}^{i+j+1}-\eta_{0}^{i+1}=\sum_{q=i+1}^{i+j} \Delta_{q} \eta_{0}
\end{aligned}
$$

there exists a constant $C_{T}$ independent of $i, j$ such that for all $t \in[0, T]$, we get

$$
\begin{gathered}
\left\|u^{i+j+1}-u^{i+1}\right\|_{B_{p, r}^{s-1}}+\left\|\eta^{i+j+1}-\eta^{i+1}\right\|_{B_{p, r}^{s-2}} \\
\leq C_{T}\left[2^{-i}+\int_{0}^{t}\left(\left\|u^{i+j}-u^{i}\right\|_{B_{p, r}^{s-1}}\right.\right. \\
\left.\left.+\left\|\eta^{i+j}-\eta^{i}\right\|_{B_{p, r}^{s-2}}\right) \mathrm{~d} \tau\right] .
\end{gathered}
$$

By induction, we obtain that

$$
\begin{aligned}
\left\|u^{i+j+1}-u^{i+1}\right\|_{L_{T}^{\infty}\left(B_{p, r}^{s-1}\right)}+\left\|\eta^{i+j+1}-\eta^{i+1}\right\|_{L_{T}^{\infty}\left(B_{p, r}^{s-2}\right)} \\
\leq \frac{\left(C_{T} T\right)^{i+1}}{(i+1) !}\left[\left\|u^{j}\right\|_{L^{\infty}\left([0, T] ; B_{p, r}^{s-1}\right)}+\left\|\eta^{j}\right\|_{L^{\infty}\left([0, T] ; B_{p, r}^{s-2}\right)}\right] \\
\quad+C_{T} \sum_{l=0}^{i} 2^{-(i-l)} \frac{\left(C_{T} T\right)^{l}}{l !} .
\end{aligned}
$$

Since $\left\|u^{j}\right\|_{L_{T}^{\infty}\left(B_{p, r}^{s}\right)},\left\|\eta^{j}\right\|_{L_{T}^{\infty}\left(B_{p, r}^{s-1}\right)}$ are bounded independently of $j$, we conclude that there exists a new constant $C_{T_{1}}$ such that

$$
\begin{aligned}
& \left\|u^{i+j+1}-u^{i+1}\right\|_{L_{T}^{\infty}\left(B_{p, r}^{s-1}\right)}+\left\|\eta^{i+j+1}-\eta^{i+1}\right\|_{L_{T}^{\infty}\left(B_{p, r}^{s-2}\right)} \\
& \leq C_{T_{1}} 2^{-i}
\end{aligned}
$$

Consequently, $\left(u^{i}, \eta^{i}\right)_{i \in \mathbb{N}}$ is a Cauchy sequence in $C([0, T]$; $\left.B_{p, r}^{s-1}\right) \times C\left([0, T] ; B_{p, r}^{s-2}\right)$. 
Step 4. We end the proof of existence of solutions.

Firstly, since $\left(u^{i}, \eta^{i}\right)_{i \in \mathbb{N}}$ is uniformly bounded in $L^{\infty}\left([0, T] ; B_{p, r}^{s}\right) \times L^{\infty}\left([0, T] ; B_{p, r}^{s-1}\right)$, according to the Fatou property for Besov space, it guarantees that $(u, \eta)$ also belongs to $L^{\infty}\left([0, T] ; B_{p, r}^{s}\right) \times L^{\infty}\left([0, T] ; B_{p, r}^{s-1}\right)$.

Secondly, for $\left(u^{i}, \eta^{i}\right)_{i \in \mathbb{N}}$ is a Cauchy sequence in $C\left([0, T] ; B_{p, r}^{s-1}\right) \times C\left([0, T] ; B_{p, r}^{s-2}\right)$, so it converges to some limit function $(u, \eta) \in C\left([0, T] ; B_{p, r}^{s-1}\right) \times C\left([0, T] ; B_{p, r}^{s-2}\right)$. An interpolation argument insures that the convergence holds in $C\left([0, T] ; B_{p, r}^{s^{\prime}}\right) \times C\left([0, T] ; B_{p, r}^{s^{\prime}-1}\right)$ for any $s^{\prime}<s$. It is easy to pass to the limit in (25) and to conclude that $(u, \eta)$ is indeed a solution to $(24)$. Thanks to the fact that $(u, \eta)$ belongs to $L^{\infty}\left([0, T] ; B_{p, r}^{s}\right) \times L^{\infty}\left([0, T] ; B_{p, r}^{s-1}\right)$ we know that the right side of the first equation in (24) belongs to $L^{\infty}\left([0, T] ; B_{p, r}^{s}\right)$, and the right side of the second equation in (24) belongs to $L^{\infty}\left([0, T] ; B_{p, r}^{s-1}\right)$. In the case of $r<\infty$, applying Lemma 13 derives $(u, \eta) \in C\left([0, T] ; B_{p, r}^{s^{\prime}}\right) \times C\left([0, T] ; B_{p, r}^{s^{\prime}-1}\right)$ for any $s^{\prime}<s$.

Finally, with (24), we obtain that $\left(u_{t}, \eta_{t}\right) \in$ $C\left([0, T] ; B_{p, r}^{s-1}\right) \times C\left([0, T] ; B_{p, r}^{s-2}\right)$ if $r<\infty$, and in $L^{\infty}\left([0, T] ; B_{p, r}^{s-1}\right) \times L^{\infty}\left([0, T] ; B_{p, r}^{s-2}\right)$ otherwise. Thus, $(u, \eta)$ $\in E_{p, r}^{s}(T) \times E_{p, r}^{s-1}(T)$. Moreover, a standard use of a sequence of viscosity approximate solutions $\left(u_{\varepsilon}, \eta_{\varepsilon}\right)_{\varepsilon>0}$ for (24) which converges uniformly in $C\left([0, T] ; B_{p, r}^{s}\right) \cap C^{1}\left([0, T] ; B_{p, r}^{s-1}\right) \times$ $C\left([0, T] ; B_{p, r}^{s-1}\right) \cap C^{1}\left([0, T] ; B_{p, r}^{s-2}\right)$ gives the continuity of solution $(u, \eta) \in E_{p, r}^{\mathcal{S}}(T) \times E_{p, r}^{\mathcal{s}-1}(T)$.

\subsection{Uniqueness and Continuity with Initial Data}

Lemma 14. Assume that $1 \leq p, r \leq \infty$, and $s>\max (1+$ $1 / p, 3 / 2)$. Let $\left(u^{1}, \eta^{1}\right)$ and $\left(u^{2}, \eta^{2}\right)$ be two given solutions to the system (24) with initial data $\left(u_{0}^{1}, \eta_{0}^{1}\right),\left(u_{0}^{2}, \eta_{0}^{2}\right) \in B_{p, r}^{s} \times$ $B_{p, r}^{s-1}$ satisfying $u^{1}, u^{2} \in L^{\infty}\left([0, T] ; B_{p, r}^{s}\right) \cap C\left([0, T] ; B_{p, r}^{s-1}\right)$ and $\eta^{1}, \eta^{2} \in L^{\infty}\left([0, T] ; B_{p, r}^{s-1}\right) \cap C\left([0, T] ; B_{p, r}^{s-2}\right)$. Then for every $t \in[0, T]$, one has

$$
\begin{aligned}
\| u^{1}- & u^{2}\left\|_{B_{p, r}^{s-1}}+\right\| \eta^{1}-\eta^{2} \|_{B_{p, r}^{s-2}} \\
\leq & \left(\left\|u_{0}^{1}-u_{0}^{2}\right\|_{B_{p, r}^{s-1}}+\left\|\eta_{0}^{1}-\eta_{0}^{2}\right\|_{B_{p, r}^{s-2}}\right) \\
& \times e^{C \int_{0}^{t}\left[\left(\left\|u^{1}\right\|_{B_{p, r}^{s}}^{s}+\left\|u^{2}\right\|_{B_{p, r}^{s}}^{s}+k+1\right)^{s_{1}+1}+\left\|\eta^{1}\right\|_{B_{p, r}^{s-1}}+\left\|\eta^{2}\right\|_{B_{p, r}^{s-1}}\right] \mathrm{d} \tau} .
\end{aligned}
$$

Proof. Denote $u^{12}=u^{2}-u^{1}, \eta^{12}=\eta^{2}-\eta^{1}$; then

$$
\begin{aligned}
& u^{12} \in L^{\infty}\left([0, T] ; B_{p, r}^{s}\right) \cap C\left([0, T] ; B_{p, r}^{s-1}\right), \\
& \eta^{12} \in L^{\infty}\left([0, T] ; B_{p, r}^{s-1}\right) \cap C\left([0, T] ; B_{p, r}^{s-2}\right),
\end{aligned}
$$

which implies that $\left(u^{12}, \eta^{12}\right) \in C\left([0, T] ; B_{p, r}^{s-1}\right) \times C([0, T]$; $\left.B_{p, r}^{s-2}\right)$, and $\left(u^{12}, \eta^{12}\right)$ satisfies the following transport equation:

$$
\begin{gathered}
\partial_{t} u^{12}+u^{1} \partial_{x} u^{12}=-u^{12} \partial_{x} u^{2}+F_{1}(t, x), \\
t>0, x \in \mathbb{R}, \\
\partial_{t} \eta^{12}+u^{1} \partial_{x} \eta^{12}=F_{0}(t, x), \quad t>0, x \in \mathbb{R}, \\
u^{12}(x, 0)=u_{0}^{12}=u_{0}^{2}-u_{0}^{1}, \quad x \in \mathbb{R}, \\
\eta^{12}(x, 0)=\eta_{0}^{12}=\eta_{0}^{2}-\eta_{0}^{1}, \quad x \in \mathbb{R},
\end{gathered}
$$

with

$$
\begin{aligned}
& F_{1}(t, x) \\
& =P(D)\left[u^{12}\left(u^{1}+u^{2}\right)+\frac{1}{2} \partial_{x} u^{12} \partial_{x}\left(u^{1}+u^{2}\right)\right. \\
& \left.+2 k u^{12}+\frac{1}{2} \eta^{12}\left(\eta^{1}+\eta^{2}+2\right)\right] \\
& +P_{1}(D)\left[-\lambda\left(\left(u^{1}\right)^{2 n+1}-\left(u^{2}\right)^{2 n+1}\right)\right. \\
& \left.+\beta\left(\left(u^{1}\right)^{2 m} u_{x x}^{1}-\left(u^{2}\right)^{2 m} u_{x x}^{2}\right)\right], \\
& F_{0}(t, x)=-u^{12} \partial_{x} \eta^{2}-\left(\eta^{12} \partial_{x} u^{1}+\eta^{2} \partial_{x} u^{12}+\partial_{x} u^{12}\right) \text {. }
\end{aligned}
$$

According to Lemma 12, we have

$$
\begin{aligned}
& e^{-C \int_{0}^{t}\left\|\partial_{x} u^{1}\right\|_{B_{p, r}^{s-1}} \mathrm{~d} \tau}\left\|u^{12}\right\|_{B_{p, r}^{s-1}} \\
& \leq\left\|u_{0}^{12}\right\|_{B_{p, r}^{s-1}} \\
& \quad+C \int_{0}^{t} e^{-C \int_{0}^{\tau}\left\|\partial_{x} u^{1}\right\|_{B_{p, r}^{s-r}} \mathrm{~d} \xi}\left(\left\|u^{12} \partial_{x} u^{2}\right\|_{B_{p, r}^{s-1}}+\left\|F_{1}\right\|_{B_{p, r}^{s-1}}\right) \mathrm{d} \tau, \\
& e^{-C \int_{0}^{t}\left\|\partial_{x} u^{1}\right\|_{B_{p, r}^{s-1}} \mathrm{~d} \tau}\left\|\eta^{12}\right\|_{B_{p, r}^{s-2}} \\
& \leq\left\|\eta_{0}^{12}\right\|_{B_{p, r}^{s-2}} \\
& \quad+C \int_{0}^{t} e^{-C \int_{0}^{\tau}\left\|\partial_{x} u^{1}\right\|_{B_{p, r}^{s-1}} \mathrm{~d} \xi}\left\|F_{0}\right\|_{B_{p, r}^{s-2}} \mathrm{~d} \tau .
\end{aligned}
$$

Similar to the proof of the third step in Section 3.1, here we only focus our attention on the high order terms. We deduce that

$$
\begin{aligned}
& \| P_{1}(D)[-\lambda\left(\left(u^{1}\right)^{2 n+1}-\left(u^{2}\right)^{2 n+1}\right) \\
&\left.+\beta\left(\left(u^{1}\right)^{2 m} u_{x x}^{1}-\left(u^{2}\right)^{2 m} u_{x x}^{2}\right)\right] \|_{B_{p, r}^{s-1}} \\
& \leq C\left(1+\left\|u^{1}\right\|_{B_{p, r}^{s}}+\left\|u^{2}\right\|_{B_{p, r}^{s}}\right)^{s_{1}+1}\left\|u^{12}\right\|_{B_{p, r}^{s-1}} .
\end{aligned}
$$


Hence,

$$
\begin{gathered}
\left\|-u^{12} \partial_{x} u^{2}+F_{1}(t, x)\right\|_{B_{p, r}^{s-1}}+\left\|F_{0}(t, x)\right\|_{B_{p, r}^{s-2}} \\
\leq\left(\left\|u^{12}\right\|_{B_{p, r}^{s-1}}+\left\|\eta^{12}\right\|_{B_{p, r}^{s-2}}\right) \\
\times\left[\left(k+1+\left\|u^{1}\right\|_{B_{p, r}^{s}}+\left\|u^{2}\right\|_{B_{p, r}^{s}}\right)^{s_{1}+1}\right. \\
\left.+\left\|\eta^{1}\right\|_{B_{p, r}^{s-1}}+\left\|\eta^{2}\right\|_{B_{p, r}^{s-1}}\right],
\end{gathered}
$$

which together with (56) makes us obtain

$$
\begin{aligned}
e^{-C \int_{0}^{t}\left\|\partial_{x} u^{1}\right\|_{B_{p, r}^{s-r}} \mathrm{~d} \tau}\left(\left\|u^{12}\right\|_{B_{p, r}^{s-1}}+\left\|\eta^{12}\right\|_{B_{p, r}^{s-2}}\right) \\
\leq\left(\left\|u_{0}^{12}\right\|_{B_{p, r}^{s-1}}+\left\|\eta_{0}^{12}\right\|_{B_{p, r}^{s-2}}\right) \\
+C \int_{0}^{t} e^{-C \int_{0}^{\tau}\left\|\partial_{x} u^{1}\right\|_{B_{p, r}^{s-r}} \mathrm{~d} \xi} \cdot\left(\left\|u^{12}\right\|_{B_{p, r}^{s-1}}+\left\|\eta^{12}\right\|_{B_{p, r}^{s-2}}\right) \\
\times\left[\left(k+1+\left\|u^{1}\right\|_{B_{p, r}^{s}}+\left\|u^{2}\right\|_{B_{p, r}^{s}}\right)^{s_{1}+1}\right. \\
\left.+\left\|\eta^{1}\right\|_{B_{p, r}^{s-1}}+\left\|\eta^{2}\right\|_{B_{p, r}^{s-1}}\right] \mathrm{d} \tau .
\end{aligned}
$$

Using Gronwall's inequality, we complete the proof of Lemma 14.

Remark 15. When $p=r=2$, the Besov space $B_{p, r}^{s}(\mathbb{R})$ coincides with the Sobolev space $H^{s}(\mathbb{R})$. Theorem 1 implies that under the assumption $\left(u_{0}, \rho_{0}-1\right) \in H^{s}(\mathbb{R}) \times H^{s-1}(\mathbb{R})$ with $s>3 / 2$, we obtain the local well-posedness for system (1), which improves the corresponding results in $[20,25,35]$ by Kato's theory, where $s \geq 2$ is assumed in $[25,35]$ and $s \geq 3$ in [20].

Remark 16. The existence time for system (1) may be chosen independently of $s$ in the following sense [43]. If $(u, \rho-$ 1) $\in C\left([0, T] ; H^{s}\right) \cap C^{1}\left([0, T] ; H^{s-1}\right) \times C\left([0, T] ; H^{s-1}\right) \cap$ $C^{1}\left([0, T] ; H^{s-2}\right)$ is the solution of system (1) with initial data $\left(u_{0}, \rho_{0}-1\right) \in H^{r} \times H^{r-1}$ for some $r>3 / 2, r \neq s$, then $(u, \rho-1) \in C\left([0, T] ; H^{r}\right) \cap C^{1}\left([0, T] ; H^{r-1}\right) \times C\left([0, T] ; H^{r-1}\right) \cap$ $C^{1}\left([0, T] ; H^{r-2}\right)$ with the same time $T$. In particular, if $(u, \rho-$ 1) $\in H^{\infty} \times H^{\infty}$, then $(u, \rho-1) \in C\left([0, T] ; H^{\infty}\right) \times$ $C\left([0, T] ; H^{\infty}\right)$

\section{Wave-Breaking Phenomena}

In this section, attention is turned to investigating conditions of wave breaking and the precise blow-up rate of strong solutions to system (1). Using Theorem 1 and Remark 16, we establish the precise wave-breaking scenarios of strong solutions to system (1).
4.1. The Proof of Theorem 2. Applying Theorem 1, Remark 16, and a simple density argument, we only need to show that Theorem 2 holds with $s \geq 3$. Here we assume $s=3$ to prove the theorem.

Noting that $\eta=\rho-1$, we rewrite the system (1) as

$$
\begin{gathered}
u_{t}-u_{x x t}+3 u u_{x}+\eta \eta_{x}+\eta_{x}+2 k u_{x} \\
+\lambda u^{2 n+1}-\beta u^{2 m} u_{x x} \\
=2 u_{x} u_{x x}+u u_{x x x}, \quad t>0, x \in \mathbb{R}, \\
\eta_{t}+u \eta_{x}+\eta u_{x}+u_{x}=0, \quad t>0, \quad x \in \mathbb{R}, \\
u(x, 0)=u_{0}(x), \quad x \in \mathbb{R}, \\
\eta(x, 0)=\eta_{0}(x)=\rho_{0}(x)-1, \quad x \in \mathbb{R} .
\end{gathered}
$$

Multiplying the first equation in (60) by $u$ and integrating by parts, we deduce that

$$
\begin{gathered}
\frac{1}{2} \frac{\mathrm{d}}{\mathrm{d} t} \int_{\mathbb{R}}\left(u^{2}+u_{x}^{2}\right) \mathrm{d} x+\int_{\mathbb{R}} \lambda u^{2 n+2} \mathrm{~d} x \\
+\int_{\mathbb{R}} \beta(2 m+1) u^{2 m} u_{x}^{2} \mathrm{~d} x \\
=\frac{1}{2} \int_{\mathbb{R}} \eta^{2} u_{x} \mathrm{~d} x+\int_{\mathbb{R}} \eta u_{x} \mathrm{~d} x .
\end{gathered}
$$

Since $\int_{\mathbb{R}} \lambda u^{2 n+2} \mathrm{~d} x \geq 0$ and $\int_{\mathbb{R}} \beta(2 m+1) u^{2 m} u_{x}^{2} \mathrm{~d} x \geq 0$, then

$$
\frac{1}{2} \frac{\mathrm{d}}{\mathrm{d} t} \int_{\mathbb{R}}\left(u^{2}+u_{x}^{2}\right) \mathrm{d} x \leq \frac{1}{2} \int_{\mathbb{R}} \eta^{2} u_{x} \mathrm{~d} x+\int_{\mathbb{R}} \eta u_{x} \mathrm{~d} x
$$

Multiplying the second equation in (60) by $\eta$ and integrating by parts, we have

$$
\frac{1}{2} \frac{\mathrm{d}}{\mathrm{d} t} \int_{\mathbb{R}} \eta^{2} \mathrm{~d} x=-\frac{1}{2} \int_{\mathbb{R}} \eta^{2} u_{x} \mathrm{~d} x-\int_{\mathbb{R}} \eta u_{x} \mathrm{~d} x,
$$

which together with (62) implies

$$
\frac{\mathrm{d}}{\mathrm{d} t} \int_{\mathbb{R}}\left(u^{2}+u_{x}^{2}+\eta^{2}\right) \mathrm{d} x \leq 0 .
$$

On the other hand, multiplying the first equation in (60) by $u_{x x}$ and integrating by parts again, we get

$$
\begin{aligned}
-\frac{1}{2} \frac{\mathrm{d}}{\mathrm{d} t} \int_{\mathbb{R}}\left(u_{x}^{2}+u_{x x}^{2}\right) \mathrm{d} x-\lambda(2 n+1) \\
\quad \times \int_{\mathbb{R}}\left(u^{2 n} u_{x}^{2}\right) \mathrm{d} x-\beta \int_{\mathbb{R}}\left(u^{2 m} u_{x x}^{2}\right) \mathrm{d} x \\
=\frac{3}{2} \int_{\mathbb{R}} u_{x}\left(u_{x}^{2}+u_{x x}^{2}\right) \mathrm{d} x \\
+\frac{1}{2} \int_{\mathbb{R}} u_{x}\left[\left(\eta^{2}\right)_{x x}+2 \eta_{x x}\right] \mathrm{d} x .
\end{aligned}
$$

Multiplying the second equation in (60) by $\eta_{x x}$ and integrating by parts, we obtain

$$
-\frac{1}{2} \frac{\mathrm{d}}{\mathrm{d} t} \int_{\mathbb{R}} \eta_{x}^{2} \mathrm{~d} x=\frac{1}{2} \int_{\mathbb{R}} u_{x}\left(\eta_{x}^{2}-2 \eta \eta_{x x}-2 \eta_{x x}\right) \mathrm{d} x,
$$


which together with (65) derives

$$
\begin{aligned}
& -\frac{1}{2} \frac{\mathrm{d}}{\mathrm{d} t} \int_{\mathbb{R}}\left(u_{x}^{2}+u_{x x}^{2}\right) \mathrm{d} x-\frac{1}{2} \frac{\mathrm{d}}{\mathrm{d} t} \int_{\mathbb{R}} \eta_{x}^{2} \mathrm{~d} x-\lambda(2 n+1) \\
& \quad \times \int_{\mathbb{R}}\left(u^{2 n} u_{x}^{2}\right) \mathrm{d} x-\beta \int_{\mathbb{R}}\left(u^{2 m} u_{x x}^{2}\right) \mathrm{d} x \\
& =\frac{3}{2} \int_{\mathbb{R}} u_{x}\left(u_{x}^{2}+u_{x x}^{2}+\eta_{x}^{2}\right) \mathrm{d} x
\end{aligned}
$$

For $\lambda(2 n+1) \int_{\mathbb{R}}\left(u^{2 n} u_{x}^{2}\right) \mathrm{d} x \geq 0$ and $\beta \int_{\mathbb{R}}\left(u^{2 m} u_{x x}^{2}\right) \mathrm{d} x \geq 0$, we have

$$
\frac{\mathrm{d}}{\mathrm{d} t} \int_{\mathbb{R}}\left(u_{x}^{2}+u_{x x}^{2}+\eta_{x}^{2}\right) \mathrm{d} x \leq-3 \int_{\mathbb{R}} u_{x}\left(u_{x}^{2}+u_{x x}^{2}+\eta_{x}^{2}\right) \mathrm{d} x
$$

Therefore, combining (64) with (68), one deduces that

$$
\begin{array}{r}
\frac{\mathrm{d}}{\mathrm{d} t} \int_{\mathbb{R}}\left(u^{2}+2 u_{x}^{2}+u_{x x}^{2}+\eta^{2}+\eta_{x}^{2}\right) \mathrm{d} x \\
\leq-3 \int_{\mathbb{R}} u_{x}\left(u_{x}^{2}+u_{x x}^{2}+\eta_{x}^{2}\right) \mathrm{d} x .
\end{array}
$$

Assume that $T \leq+\infty$ and there exists $M>0$ such that

$$
\begin{gathered}
u_{x}(t, x) \geq-M, \quad \text { for }(t, x) \in[0, T) \times \mathbb{R}, \\
\left\|\rho_{x}(t, \cdot)\right\|_{L^{\infty}} \leq M, \quad \text { for } t \in[0, T) .
\end{gathered}
$$

It follows from (69) that

$$
\begin{gathered}
\frac{\mathrm{d}}{\mathrm{d} t} \int_{\mathbb{R}}\left(u^{2}+2 u_{x}^{2}+u_{x x}^{2}+\eta^{2}+\eta_{x}^{2}\right) \mathrm{d} x \\
\leq 3 M \int_{\mathbb{R}}\left(u_{x}^{2}+u_{x x}^{2}+\eta_{x}^{2}\right) \mathrm{d} x .
\end{gathered}
$$

Applying Gronwall's inequality to (71) yields, for all $t \in[0, T)$,

$$
\|u(t)\|_{H^{2}}^{2}+\|\eta(t)\|_{H^{1}}^{2} \leq 2\left(\left\|u_{0}\right\|_{H^{2}}^{2}+\left\|\eta_{0}\right\|_{H^{1}}^{2}\right) e^{3 M T}
$$

Differentiating the first equation in (60) with respect to $x$ and multiplying the obtained equation by $u_{x x x}$, then integrating by parts, we get

$$
\begin{aligned}
\frac{1}{2} \frac{\mathrm{d}}{\mathrm{d} t} \int_{\mathbb{R}} & \left(u_{x x}^{2}+u_{x x x}^{2}\right) \mathrm{d} x \\
= & \frac{\lambda(2 n-1) 2 n(2 n+1)}{3} \\
& \times \int_{\mathbb{R}} u^{2 n-2} u_{x}^{4} \mathrm{~d} x-\lambda(2 n+1) \int_{\mathbb{R}} u^{2 n} u_{x x}^{2} \mathrm{~d} x \\
& +\beta m(2 m-1) \int_{\mathbb{R}} u^{2 m-2} u_{x x}^{2} u_{x}^{2} \mathrm{~d} x \\
& +\beta m \int_{\mathbb{R}} u_{x x}^{3} u^{2 m-1} \mathrm{~d} x-\beta \int_{\mathbb{R}} u^{2 m} u_{x x x}^{2} \mathrm{~d} x-\frac{15}{2} \\
& \times \int_{\mathbb{R}} u_{x} u_{x x}^{2} \mathrm{~d} x-\frac{5}{2} \int_{\mathbb{R}} u_{x} u_{x x x}^{2} \mathrm{~d} x \\
& -2 \int_{\mathbb{R}} \eta_{x} \eta_{x x} u_{x x} \mathrm{~d} x+\int_{\mathbb{R}} \eta \eta_{x x} u_{x x x} \mathrm{~d} x \\
& +\int_{\mathbb{R}} \eta_{x x} u_{x x x} \mathrm{~d} x .
\end{aligned}
$$

Differentiating the second equation twice in (60) with respect to $x$ and multiplying the resulted equation by $\eta_{x x}$, then integrating by parts, we deduce that

$$
\begin{aligned}
\frac{1}{2} \frac{\mathrm{d}}{\mathrm{d} t} \int_{\mathbb{R}} \eta_{x x}^{2} \mathrm{~d} x= & -\frac{5}{2} \int_{\mathbb{R}} u_{x} \eta_{x x}^{2} \mathrm{~d} x-3 \int_{\mathbb{R}} \eta_{x} \eta_{x x} u_{x x} \mathrm{~d} x \\
& -\int_{\mathbb{R}} \eta \eta_{x x} u_{x x x} \mathrm{~d} x-\int_{\mathbb{R}} \eta_{x x} u_{x x x} \mathrm{~d} x
\end{aligned}
$$

which together with (73), $\lambda(2 n+1) \int_{\mathbb{R}} u^{2 n} u_{x x}^{2} \mathrm{~d} x \geq 0$, and $\beta \int_{\mathbb{R}} u^{2 m} u_{x x x}^{2} \mathrm{~d} x \geq 0$ one has

$$
\begin{aligned}
\frac{1}{2} \frac{\mathrm{d}}{\mathrm{d} t} \int_{\mathbb{R}} & \left(u_{x x}^{2}+u_{x x x}^{2}+\eta_{x x}^{2}\right) \mathrm{d} x \\
\leq & 10 M \int_{\mathbb{R}}\left(u_{x x}^{2}+u_{x x x}^{2}+\eta_{x x}^{2}\right) \mathrm{d} x \\
& +\frac{\lambda(2 n-1) 2 n(2 n+1)}{3} \\
& \times\left\|u_{x}\right\|_{L^{\infty}}^{2}\|u\|_{L^{\infty}}^{2 n-2} \\
& \times \int_{\mathbb{R}} u_{x}^{2} \mathrm{~d} x+\beta m(2 m-1)\left\|u_{x}\right\|_{L^{\infty}}^{2}\|u\|_{L^{\infty}}^{2 m-2} \\
& \times \int_{\mathbb{R}} u_{x x}^{2} \mathrm{~d} x+\beta m\|u\|_{L^{\infty}}^{2 m-1} \int_{\mathbb{R}} u_{x x}^{3} \mathrm{~d} x,
\end{aligned}
$$

where we have used (64), (70), and (72).

Applying Sobolev's embedding theorem and Holder's inequality yields

$$
\begin{aligned}
\int_{\mathbb{R}} u_{x x}^{3} \mathrm{~d} x & \leq\left\|u_{x x}\right\|_{L^{\infty}}^{2} \int_{\mathbb{R}}\left|u_{x x}\right| \mathrm{d} x \\
& \leq\left\|u_{x x}\right\|_{H^{(1 / 2)+}}^{2} \int_{\mathbb{R}}\left|u_{x x}\right| \mathrm{d} x \leq\|u\|_{H^{3}}^{2}\|u\|_{H^{2}},
\end{aligned}
$$


together with (72) and (75); thus there exists $C_{1}>0$ and $C_{2}>$ 0 such that

$$
\begin{aligned}
& \frac{\mathrm{d}}{\mathrm{d} t} \int_{\mathbb{R}}\left(u_{x x}^{2}+u_{x x x}^{2}+\eta_{x x}^{2}\right) \mathrm{d} x \\
& \quad \leq C_{1}+C_{2} \int_{\mathbb{R}}\left(u_{x x}^{2}+u_{x x x}^{2}+\eta_{x x}^{2}\right) \mathrm{d} x .
\end{aligned}
$$

Using Gronwall's inequality, for all $t \in(0, T)$, we have

$$
\begin{aligned}
& \int_{\mathbb{R}}\left(u_{x x}^{2}+u_{x x \mathrm{x}}^{2}+\eta_{x x}^{2}\right) \mathrm{d} x \\
& \quad \leq e^{C_{2} t} \int_{\mathbb{R}}\left(u_{0 x x}^{2}+u_{0 x x x}^{2}+\eta_{0 x x}^{2}\right) \mathrm{d} x+\frac{C_{1}}{C_{2}}\left(e^{C_{2} t}-1\right),
\end{aligned}
$$

which contradicts the assumption of the maximal existence time $T<+\infty$.

Conversely, using Sobolev's embedding theorem $H^{s} \hookrightarrow$ $L^{\infty}(s>1 / 2)$, we derive that if condition (9) in Theorem 2 holds, the corresponding solution blows up in finite time, which completes the proof of Theorem 2.

4.2. The Proof of Theorem 3. Firstly, we present three lemmas which are used to prove the blow-up mechanisms and the global existence of solutions to system (1).

Lemma 17 (see $[44])$. Let $T>0$ and $u \in C^{1}\left([0, T) ; H^{2}(\mathbb{R})\right)$. Then for all $t \in[0, T)$, there exists at least one point $\xi(t) \in \mathbb{R}$ with

$$
m(t)=\inf _{x \in \mathbb{R}} u_{x}(t, x)=u_{x}(t, \xi(t)) .
$$

The function $m(t)$ is absolutely continuous on $(0, T)$ with

$$
\frac{\mathrm{d}}{\mathrm{dt}} m(t)=u_{x t}(t, \xi(t)) \quad \text { a.e. on }(0, T) \text {. }
$$

Now consider the following trajectory equation:

$$
\begin{gathered}
\frac{\mathrm{d}}{\mathrm{dt}} q(t, x)=u(t, q(t, x)), \quad t \in[0, T), \\
q(x, 0)=x, \quad x \in \mathbb{R},
\end{gathered}
$$

where $u$ denotes the first component of solution $(u, \rho-1)$ to system (1).

Lemma 18 (see [45]). Let $u \in C\left([0, T) ; H^{s}(\mathbb{R})\right) \quad \cap$ $C^{1}\left([0, T) ; H^{s-1}(\mathbb{R})\right)$ with $s \geq 2$. Then, (81) has a unique solution: $q \in C^{1}([0, T) \times \mathbb{R}, \mathbb{R})$. Moreover, the map $q(t, \cdot)$ is an increasing diffeomorphism of $\mathbb{R}$ for every $t \in[0, T)$ and

$$
q_{x}(t, x)=e^{\int_{0}^{t} u_{x}(\tau, q(\tau, x)) \mathrm{d} \tau}>0, \quad \text { for all }(t, x) \in[0, T) \times \mathbb{R} .
$$

Lemma 19 (see [23]). Let $\left(u_{0}, \rho_{0}-1\right) \in H^{s} \times H^{s-1}$ with $s \geq 2$, and let $T>0$ be the maximal existence time of the corresponding solution $(u, \rho-1)$ to system (1). Thus,

$$
\rho(t, q(t, x)) q_{x}(t, x)=\rho_{0}(x), \quad \text { for all }(t, x) \in[0, T) \times \mathbb{R} \text {. }
$$

Proof of Theorem 3. The technique used here is inspired from [20]. Similar to the proof of Theorem 2, we only need to show that Theorem 3 holds with some $s=3$.

By Lemma 17, we obtain that there exists $\xi(t)$ with $t \in$ $[0, T)$ such that

$$
m(t)=u_{x}(t, \xi(t))=\inf _{x \in \mathbb{R}} u_{x}(t, x), \quad \text { for all } t \in[0, T) .
$$

Hence, we have $u_{x x}(t, \xi(t))=0$ for a.e. $t \in[0, T)$.

On the other hand, since $q(t, \cdot): \mathbb{R} \rightarrow \mathbb{R}$ is diffeomorphism for any $t \in[0, T)$, there exists $x_{1}(t) \in \mathbb{R}$ such that $q\left(t, x_{1}(t)\right)=\xi(t)$, for all $t \in[0, T)$. Using the second equation in system (1) and the trajectory equation (81), we have

$$
\frac{\mathrm{d}}{\mathrm{d} t} \rho(t, q(t, x))=-u_{x}(t, q(t, x)) \rho(t, q(t, x)) .
$$

Hence,

$$
\rho(t, q(t, x))=\rho_{0}(x) e^{-\int_{0}^{t} u_{x}(\tau, q(\tau, x)) \mathrm{d} \tau} .
$$

Taking $x=x_{1}(t)$ in (85), together with $q\left(t, x_{1}(t)\right)=\xi(t)$, for all $t \in[0, T)$, then

$$
\frac{\mathrm{d}}{\mathrm{d} t} \rho(t, \xi(t))=-u_{x}(t, \xi(t)) \rho(t, \xi(t)) .
$$

Using the assumption $\rho_{0}\left(x_{0}\right)=0$ with the point $x_{0}$ defined by $u_{0 x}\left(x_{0}\right)=\inf _{x \in \mathbb{R}} u_{0 x}(x)$ in Theorem 3 and letting $\xi(0)=x_{0}$, we deduce that $\rho_{0}(\xi(0))=\rho_{0}\left(x_{0}\right)=0$. From (87), we get

$$
\rho(t, \xi(t))=0, \quad \text { for all } t \in[0, T) .
$$

If $g(x)=(1 / 2) e^{-|x|}, x \in \mathbb{R}$, then $\left(1-\partial_{x x}\right)^{-1} f=g * f$ for all $f \in L^{2}(\mathbb{R})$, so we have $\partial_{x}^{2}\left(1-\partial_{x x}\right)^{-1} f=\partial_{x}^{2} g * f=g * f-f$. For $m=0$, differentiating the first equation in system (7) with respect to the variable $x$

$$
\begin{aligned}
u_{t}+u u_{x}= & P(D)\left[u^{2}+\frac{u_{x}^{2}}{2}+2 k u+\frac{\rho^{2}}{2}\right] \\
& +P_{1}(D)\left[-\lambda u^{2 n+1}+\beta u_{x x}\right]
\end{aligned}
$$

yields

$$
\begin{aligned}
u_{t x}= & -\frac{1}{2} u_{x}^{2}-u u_{x x}+u^{2}+2 k u+\frac{1}{2} \rho^{2} \\
& -g *\left(u^{2}+\frac{1}{2} u_{x}^{2}+2 k u+\frac{1}{2} \rho^{2}\right) \\
& -\lambda \partial_{x}\left(g * u^{2 n+1}\right)+\beta \partial_{x}(g * u)-\beta u_{x} .
\end{aligned}
$$

Note inequality $(1 / 2) u^{2}(x) \leq g *\left(u^{2}+(1 / 2) u_{x}^{2}\right)(x)$ (see page $347,(5.8)$ in [28]). Using Sobolev's embedding theorem and (64), we obtain

$$
\begin{aligned}
u^{2}(t, x) & =\int_{-\infty}^{x} u u_{x} \mathrm{~d} x-\int_{x}^{+\infty} u u_{x} \mathrm{~d} x \leq \int_{\mathbb{R}}\left|u u_{x}\right| \mathrm{d} x \\
& \leq \frac{1}{2} \int_{\mathbb{R}}\left(u^{2}+u_{x}^{2}\right) \mathrm{d} x \leq \frac{1}{2}\left(\left\|u_{0}\right\|_{H^{1}}^{2}+\left\|\eta_{0}\right\|_{L^{2}}^{2}\right) .
\end{aligned}
$$


Here we denote $C_{12}=(\sqrt{2} / 2)\left(\left\|u_{0}\right\|_{H^{1}}^{2}+\left\|\eta_{0}\right\|_{L^{2}}^{2}\right)^{1 / 2}$, and thus $\|u(t, \cdot)\|_{L^{\infty}} \leq C_{12}$.

Denoting $C_{11}=\left(\left\|u_{0}\right\|_{H^{1}}^{2}+\left\|\eta_{0}\right\|_{L^{2}}^{2}\right)^{1 / 2}$ and combining with (64) yield that $\|u\|_{H^{1}} \leq C_{11}$. Together with the definition of $m(t),(88)$, and $u_{x x}(t, \xi(t))=0$ for a.e. $t \in[0, T)$, from $(90)$ we derive that

$$
\begin{aligned}
\frac{\mathrm{d} m(t)}{\mathrm{d} t} \leq & -\frac{1}{2} m^{2}(t)-\beta m(t) \\
& +\left[\frac{u^{2}}{2}+2 k u-g *(2 k u)\right. \\
& \left.\quad-\lambda \partial_{x}\left(g * u^{2 n+1}\right)+\beta \partial_{x}(g * u)\right](t, \xi(t)) .
\end{aligned}
$$

Using Young's inequality, we have for all $t \in[0, T)$,

$$
\begin{gathered}
\|g * u\|_{L^{\infty}} \leq\|g\|_{L^{1}}\|u\|_{L^{\infty}} \leq C_{12}, \\
\left|\partial_{x}\left(g * u^{2 n+1}\right)\right|=\left|g_{x} * u^{2 n+1}\right| \\
\leq\left\|g_{x}\right\|\left\|_{L^{2}}\right\| u^{2 n+1} \|_{L^{2}} \leq \frac{1}{2}\left(C_{12}\right)^{2 n+1}, \\
\left|\partial_{x}(g * u)\right| \leq\left\|g_{x}\right\|_{L^{1}}\|u\|_{L^{\infty}} \leq C_{12},
\end{gathered}
$$

which together with (92) makes us deduce that

$$
\frac{\mathrm{d} m_{1}(t)}{\mathrm{d} t} \leq-\frac{1}{2} m_{1}^{2}(t)+K
$$

where

$$
\begin{gathered}
m_{1}(t)=m(t)+\beta, \\
K=\frac{1}{2\left(C_{12}\right)^{2}}+(4 k+\beta) C_{12}+\frac{1}{2} \lambda\left(C_{12}\right)^{2 n+1}+\frac{1}{2} \beta^{2} .
\end{gathered}
$$

By the assumption of $m_{1}(0)=u_{0 x}\left(x_{0}\right)+\beta<-\sqrt{2 K}$, we have $m_{1}^{2}(0)>2 K$. We now claim that $m_{1}(t)<-\sqrt{2 K}$ is true for any $t \in[0, T)$. In fact, assume that $m_{1}(t) \geq-\sqrt{2 K}$ for some $t \in[0, T)$; since $m_{1}(t)$ is continuous, we deduce that there exists $t_{0} \in(0, T)$ such that $m_{1}^{2}(t)>2 K$ for $t \in\left[0, t_{0}\right)$, but $m_{1}^{2}\left(t_{0}\right)=2 K$. Combining this with (94) gives $\mathrm{d} m_{1}(t) / \mathrm{d} t<0$ a.e. on $\left[0, t_{0}\right)$. Since $m_{1}(t)$ is absolutely continuous on $\left[0, t_{0}\right]$, so we get the contradiction $m_{1}\left(t_{0}\right)<m_{1}(0)=u_{0 x}\left(x_{0}\right)+\beta<$ $-\sqrt{2 K}$. Thus we prove the claim.

Now we deduce that $m_{1}(t)$ is strictly decreasing on $[0, T)$. Choosing that $\delta \in(0,1)$ such that $-\sqrt{\delta} m_{1}(0)=\sqrt{2 K}$, then we obtain from (94) that

$$
\begin{array}{r}
\frac{\mathrm{d} m_{1}(t)}{\mathrm{d} t} \leq-\frac{1}{2} m_{1}^{2}(t)+\frac{\delta}{2} m_{1}^{2}(0) \leq-\frac{1-\delta}{2} m_{1}^{2}(t) \\
\text { a.e. on }[0, T) .
\end{array}
$$

For $m_{1}(t)$ is locally Lipschitz on $[0, T)$ and strictly negative, we get $1 / m_{1}(t)$ is also locally Lipschitz on $[0, T)$. This gives

$$
\frac{\mathrm{d}}{\mathrm{d} t}\left(\frac{1}{m_{1}(t)}\right)=-\frac{1}{m_{1}^{2}(t)} \frac{\mathrm{d} m_{1}(t)}{\mathrm{d} t} \geq \frac{1-\delta}{2} \quad \text { a.e. on }[0, T) .
$$

Integration of this inequality yields

$$
-\frac{1}{m_{1}(t)}+\frac{1}{m_{1}(0)} \leq-\frac{1-\delta}{2} t \text { a.e. on }[0, T) \text {. }
$$

Since $m_{1}(t)<0$ on $[0, T)$, we obtain that the maximal existence time $T \leq-2 /(1-\delta) m_{1}(0)<\infty$. Moreover, thanks to $m_{1}(0)=u_{0 x}\left(x_{0}\right)+\beta<0$ again, we get that

$$
\begin{aligned}
u_{x}(t, \xi(t)) \leq & \frac{2\left(u_{0 x}\left(x_{0}\right)+\beta\right)}{2+t(1-\delta)\left(u_{0 x}\left(x_{0}\right)+\beta\right)}-\beta \\
& \longrightarrow-\infty\left(t \longrightarrow \frac{-2}{(1-\delta)\left(u_{0 x}\left(x_{0}\right)+\beta\right)}\right),
\end{aligned}
$$

which completes the proof of Theorem 3.

Remark 20. From (81) and (85), one deduces that if $\left(u_{0}, \rho_{0}\right) \in$ $H^{s} \times H^{s-1}$ with $s>3 / 2$ and the component $u(t, x)$ does not break in finite time $T$, then the component $\rho$ is uniformly bounded for all $(t, x) \in[0, T) \times \mathbb{R}$. In fact, if there exists $M>0$ such that $u_{x}(t, x) \geq-M$, for all $(t, x) \in[0, T) \times \mathbb{R}$, thus

$$
\begin{aligned}
\|\rho(t)\|_{L^{\infty}} & =\|\rho(t, q(t, \cdot))\|_{L^{\infty}} \\
& =\left\|e^{-\int_{0}^{t} u_{x}(\tau, \cdot) \mathrm{d} \tau} \rho_{0}(\cdot)\right\|_{L^{\infty}} \leq e^{M T}\left\|\rho_{0}\right\|_{L^{\infty}}, \\
& \text { for all } t \in[0, T) .
\end{aligned}
$$

We find that there is a similar estimation in [25].

Remark 21. Because of the presence of high order term $u^{2 m} u_{x x}$ in system (1), it is difficult to obtain the estimate for the term $-\beta u^{2 m} u_{x}$ without the assumption of $m=0$.

4.3. The Proof of Theorem 4. Similar to the proof of Theorem 2, here we only need to show that Theorem 4 holds with $s=3$. Note that system (1) with $k=0$ is invariant under the transformation $(u, x) \rightarrow(-u,-x)$ and $(\rho, x) \rightarrow(\rho,-x)$. Thus, if $u_{0}$ is odd and $\rho_{0}$ is even, then the corresponding solution $(u(t, x), \rho(t, x))$ satisfies that $u(t, x)$ is odd and $\rho(t, x)$ is even with respect to $x$ for all $t \in(0, T)$, where $T$ is the maximal existence time. Hence, $u(t, 0)=0, \rho_{x}(t, 0)=0$.

Thanks to the transport equation $\rho_{t}+u \rho_{x}+\rho u_{x}=0$ at the point $x=0$, we have

$$
\begin{gathered}
\frac{\mathrm{d}}{\mathrm{d} t} \rho(t, 0)+\rho(t, 0) \partial_{x} u(t, 0)=0, \\
\rho(0,0)=0,
\end{gathered}
$$

which derives that $\rho(t, 0)=0$. Noting that $u^{2 m} u_{x x}=$ $\partial_{x}\left[u^{2 m} u_{x}\right]-2 m u^{2 m-1}\left(u_{x}\right)^{2}$, we obtain

$$
\begin{aligned}
\partial_{x} g * & \left(u^{2 m} u_{x x}\right) \\
& =\partial_{x}\left(1-\partial_{x x}\right)^{-1}\left[\partial_{x}\left(u^{2 m} u_{x}\right)-2 m u^{2 m-1} u_{x}^{2}\right] \\
& =g *\left(u^{2 m} u_{x}\right)-u^{2 m} u_{x}-2 m \partial_{x}\left[g *\left(u^{2 m-1} u_{x}^{2}\right)\right] .
\end{aligned}
$$


For $k=0$, differentiating the first equation in system (7) with respect to the variable $x$

$$
\begin{aligned}
u_{t}+u u_{x}= & -\partial_{x}\left(1-\partial_{x x}\right)^{-1}\left[u^{2}+\frac{u_{x}^{2}}{2}+\frac{\rho^{2}}{2}\right] \\
& +\left(1-\partial_{x x}\right)^{-1}\left[-\lambda u^{2 n+1}+\beta u^{2 m} u_{x x}\right]
\end{aligned}
$$

yields

$$
\begin{aligned}
u_{t x}= & -\frac{1}{2} u_{x}^{2}-u u_{x x}+u^{2}+\frac{1}{2} \rho^{2} \\
& -g *\left(u^{2}+\frac{1}{2} u_{x}^{2}+\frac{1}{2} \rho^{2}\right)-\lambda \partial_{x}\left(g * u^{2 n+1}\right) \\
& +\beta g *\left(u^{2 m} u_{x}\right)-\beta u^{2 m} u_{x}-2 m \beta \partial_{x}\left[g *\left(u^{2 m-1} u_{x}^{2}\right)\right] .
\end{aligned}
$$

Let $M(t)=u_{x}(t, 0)$, and we have

$$
\begin{aligned}
\frac{\mathrm{d} M(t)}{\mathrm{d} t} \leq- & \frac{1}{2} M^{2}(t) \\
+ & {\left[-\lambda \partial_{x}\left(g * u^{2 n+1}\right)+\beta g *\left(u^{2 m} u_{x}\right)\right.} \\
& \left.-2 m \beta \partial_{x}\left(g *\left(u^{2 m-1} u_{x}^{2}\right)\right)\right](t, 0) .
\end{aligned}
$$

Denoting $C_{11}=\left(\left\|u_{0}\right\|_{H^{1}}^{2}+\left\|\eta_{0}\right\|_{L^{2}}^{2}\right)^{1 / 2}$ and combining with (64) yield that $\|u\|_{H^{1}} \leq C_{11}$. By using Young's inequality, we deduce for all $(t, x) \in[0, T) \times \mathbb{R}$ that

$$
\begin{aligned}
\left|g *\left(u^{2 m} u_{x}\right)\right| & \leq\|g\|_{L^{2}}\left\|u^{2 m} u_{x}\right\|_{L^{2}} \\
& \leq \frac{1}{2}\|u\|_{L^{\infty}}^{2 m}\|u\|_{H^{1}}=\frac{1}{2} C_{12}^{2 m} C_{11}, \\
\left|\partial_{x}\left(g *\left(u^{2 m-1} u_{x}^{2}\right)\right)\right| & \leq\left\|g_{x}\right\|_{L^{\infty}}\left\|u^{2 m-1} u_{x}^{2}\right\|_{L^{1}} \\
& \leq \frac{1}{2}\|u\|_{L^{\infty}}^{2 m-1}\|u\|_{H^{1}}^{2} \leq \frac{1}{2} C_{12}^{2 m-1} C_{11}^{2},
\end{aligned}
$$

which together with (93) and (105) derives

$$
\frac{\mathrm{d} M(t)}{\mathrm{d} t} \leq-\frac{1}{2} M^{2}(t)+K_{1}
$$

where

$$
K_{1}=\frac{1}{2} \lambda\left(C_{12}\right)^{2 n+1}+\frac{1}{2} \beta C_{12}^{2 m} C_{11}+\beta m C_{12}^{2 m-1} C_{11}^{2} .
$$

Similarly as in the proof of Theorem 3, from (108) one gets that $M(t)<M(0)<-\sqrt{2 K_{1}}$ for all $t \in[0, T)$, and there exists $\delta_{1} \in(0,1)$ such that $-\sqrt{\delta_{1}} M(0)=\sqrt{2 K_{1}}$. Thus,

$$
-\frac{1}{M(t)}+\frac{1}{M(0)} \leq-\frac{1-\delta_{1}}{2} t \text { a.e. on }[0, T) \text {, }
$$

which implies the maximal existence time $T_{1} \leq-2 /(1-$ $\left.\delta_{1}\right) M(0)<\infty$. Then,

$$
\begin{aligned}
& u_{x}(t, 0) \leq \frac{2 u_{0 x}(0)}{2+t\left(1-\delta_{1}\right) u_{0 x}(0)} \longrightarrow-\infty \\
& \text { as } t \longrightarrow-\frac{2}{\left(1-\delta_{1}\right) u_{0 x}(0)},
\end{aligned}
$$

which completes the first part of the proof of Theorem 4.
Differentiating the transport equation $\rho_{t}+u \rho_{x}+\rho u_{x}=$ 0 with respect to the variable $x$, together with the trajectory equation (81), we get

$$
\begin{aligned}
\frac{\mathrm{d} \rho_{x}(t, q(t, x))}{\mathrm{d} t}= & -u_{x x}(t, q(t, x)) \rho(t, q(t, x)) \\
& -2 u_{x}(t, q(t, x)) \rho_{x}(t, q(t, x)) .
\end{aligned}
$$

Taking $x=x_{1}(t)$ and noting that $q\left(t, x_{1}(t)\right)=\xi(t)$ and $u_{x x}(t, \xi(t))=0$, we have

$$
\frac{\mathrm{d} \rho_{x}(t, \xi(t))}{\mathrm{d} t}=-2 u_{x}(t, \xi(t)) \rho_{x}(t, \xi(t))
$$

Noting the assumption, (84), and $\xi(0)=x_{0}, \rho_{0 x}(\xi(0))=$ $\rho_{0 x}\left(x_{0}\right)$ yields that

$$
\begin{aligned}
\rho_{x}(t, \xi(t)) & =\rho_{0 x}\left(x_{0}\right) e^{-2 \int_{0}^{t} u_{x}(\tau, \xi(\tau)) \mathrm{d} \tau} \\
& =\rho_{0 x}\left(x_{0}\right) e^{-2 \int_{0}^{t} \inf _{x \in \mathbb{R}} u_{x}(\tau, x) \mathrm{d} \tau} .
\end{aligned}
$$

Thanks to (111), for any $t \in[0, T)$, we have

$$
\begin{aligned}
e^{-2 \int_{0}^{t} \inf _{x \in \mathbb{R}} u_{x}(\tau, x) \mathrm{d} \tau} & \geq e^{-2 \int_{0}^{t}\left[2 u_{0 x}(0) /\left(2+\tau\left(1-\delta_{1}\right) u_{0 x}(0)\right)\right] \mathrm{d} \tau} \\
& =e^{\left(-4 /\left(1-\delta_{1}\right)\right) \ln \left[1+\left(\left(1-\delta_{1}\right) u_{0 x}(0) / 2\right) t\right]}
\end{aligned}
$$

Note that $e^{\left(-4 /\left(1-\delta_{1}\right)\right) \ln \left[1+\left(\left(1-\delta_{1}\right) u_{0 x}(0) / 2\right) t\right]} \rightarrow \quad+\infty$ as $t \quad \rightarrow$ $-2 /\left(1-\delta_{1}\right) u_{0 x}(0)$.

Therefore, if $\rho_{0 x}\left(x_{0}\right)>0$, from (115), for $0<T_{2} \leq-2 /(1-$ $\left.\delta_{1}\right) u_{0 x}(0)$, we have

$$
\sup _{x \in \mathbb{R}} \rho_{x}(t, x) \geq \rho_{x}(t, \xi(t)) \longrightarrow+\infty \quad \text { as } t \longrightarrow T_{2}^{-} \text {. }
$$

On the other hand, if $\rho_{0 x}\left(x_{0}\right)<0$, for $0<T_{2} \leq-2 /(1-$ $\left.\delta_{1}\right) u_{0 x}(0)$, it follows from (115) that

$$
\inf _{x \in \mathbb{R}} \rho_{x}(t, x) \leq \rho_{x}(t, \xi(t)) \longrightarrow-\infty \quad \text { as } t \longrightarrow T_{2}^{-} .
$$

Now we complete the proof of Theorem 4.

Remark 22. Under the same conditions in Theorem 3 with $s>5 / 2$ and $\rho_{0 x}\left(x_{0}\right) \neq 0$, we follow a similar argument of Theorem 4 to obtain the same blow-up results (i) and (ii) in Theorem 4.

Remark 23. Thanks to the assumption of $k=0$, system (1) is invariant under the transformation $(u, x) \rightarrow(-u,-x)$ and $(\rho, x) \rightarrow(\rho,-x)$.

4.4. Blow-Up Rate. Having established the wave-breaking results for system (1), we give the estimate for the blow-up rate of solutions to system (1). 
Proof of Theorem 5. Noting that $|g * \eta| \leq(1 / 2)\left(\|g\|_{L^{2}}^{2}+\|\right.$ $\left.\eta \|_{L^{2}}^{2}\right) \leq(1 / 2)\left(1+\left\|u_{0}\right\|_{H^{1}}^{2}+\left\|\eta_{0}\right\|_{L^{2}}^{2}\right)$, we have

$$
\begin{aligned}
0 & \leq g *\left(u^{2}+\frac{1}{2} u_{x}^{2}+\frac{1}{2} \rho^{2}\right) \\
& \leq\|g\|_{L^{\infty}}\left\|u^{2}+\frac{1}{2} u_{x}^{2}+\frac{1}{2} \eta^{2}\right\|_{L^{1}}+|g * \eta|+\frac{1}{2} \\
& \leq 1+\left\|u_{0}\right\|_{H^{1}}^{2}+\left\|\eta_{0}\right\|_{L^{2}}^{2}=1+2 C_{12}^{2} .
\end{aligned}
$$

Similar to the proof of Theorem 3, we obtain

$$
\frac{\mathrm{d} m_{1}(t)}{\mathrm{d} t} \leq-\frac{1}{2} m_{1}^{2}(t)+K_{2}
$$

where

$$
K_{2}=1+3\left(C_{12}\right)^{2}+(4 k+\beta) C_{12}+\frac{1}{2} \lambda\left(C_{12}\right)^{2 n+1}+\frac{1}{2} \beta^{2} .
$$

Therefore, choosing $\varepsilon \in(0,1 / 2)$ and using (99), we find $t_{0} \in(0, T)$ such that $m_{1}\left(t_{0}\right)<-\sqrt{2 K_{2}+K_{2} / \varepsilon}$. Since $m_{1}(t)$ is locally Lipschitz, it follows that $m_{1}(t)$ is absolutely continuous. Integrating (119) on interval $\left[t_{0}, t\right)$ with $t \in$ $\left[t_{0}, T\right)$, we deduce that $m_{1}(t)$ is decreasing on $\left[t_{0}, T\right)$ and

$$
m_{1}(t)<-\sqrt{2 K_{2}+\frac{K_{2}}{\varepsilon}}<-\sqrt{\frac{K_{2}}{\varepsilon}}, \quad t \in\left[t_{0}, T\right) .
$$

It deduces from (119) that

$$
\begin{array}{r}
\left|\frac{\mathrm{d} m_{1}(t)}{\mathrm{d} t}+\frac{1}{2} m_{1}^{2}(t)\right| \leq K_{2} \Longrightarrow-K_{2}-\frac{1}{2} m_{1}^{2}(t) \\
\leq \frac{\mathrm{d} m_{1}(t)}{\mathrm{d} t} \leq K_{2}-\frac{1}{2} m_{1}^{2}(t) \\
\text { a.e. } t \in\left(t_{0}, T\right) .
\end{array}
$$

Noting that $m_{1}(t)<0$ on $\left(t_{0}, T\right)$, we obtain

$$
\frac{1}{2}-\varepsilon \leq \frac{\mathrm{d}}{\mathrm{d} t}\left(\frac{1}{m_{1}(t)}\right) \leq \frac{1}{2}+\varepsilon \text { for a.e. } t \in\left(t_{0}, T\right) \text {. }
$$

Integrating the above relation on $(t, T)$ with $t \in\left[t_{0}, T\right)$ and noting that

$$
\lim _{t \rightarrow T^{-}} m_{1}(t)=-\infty
$$

we have $((1 / 2)-\varepsilon)(T-t) \leq-\left(1 / m_{1}(t)\right) \leq((1 / 2)+\varepsilon)(T-t)$.

Since $\varepsilon \in(0,1 / 2)$ is arbitrary, it deduces from (95) and (124) that

$$
\lim _{t \rightarrow T^{-}}\left[\inf _{x \in \mathbb{R}}\left(u_{x}(t, x)+\beta\right)(T-t)\right]=-2,
$$

which completes the proof of Theorem 5 .

Remark 24. Let $T<\infty$ be the blow-up time of the corresponding solution to system (1). The initial data $\left(u_{0}, \rho_{0}-\right.$ 1) $\in H^{s} \times H^{s-1}$ with $s>3 / 2$ satisfies the assumptions of Theorem 4 . Then,

$$
\lim _{t \rightarrow T^{-}}\left[\inf _{x \in \mathbb{R}} u_{x}(t, 0)(T-t)\right]=-2 .
$$

Proof. The argument is similar to the proof of Theorem 5; here we omit it.

\section{The Proof of Theorem 6}

In this section, we first give a lemma on global strong solutions to system (1). Then, we present the proof of Theorem 6. We need to pay more attention to the assumption $m=0$ and $\rho_{0}(x) \neq 0$ for all $x \in \mathbb{R}$.

Lemma 25. Assume $m=0$ in system (1) and $\left(u_{0}, \rho_{0}-1\right) \epsilon$ $H^{s} \times H^{s-1}$ with $s>3 / 2$. Let $T>0$ be the maximal existence time of corresponding solution $(u, \rho)$ to system $(1)$ in the case of $m=0$ with initial data $\left(u_{0}, \rho_{0}\right)$. If $\rho_{0}(x) \neq 0$ for all $x \in \mathbb{R}$, then there exists a constant $\beta_{2}>0$ such that

$$
\begin{aligned}
& \lim _{t \rightarrow T^{-} x \in \mathbb{R}} \inf _{x}(t, x) \\
& \quad \geq-\frac{1}{2 \beta_{2}}\left[3+2 \beta^{2}+2\left\|\left(u_{0}, \rho_{0}-1\right)\right\|_{H^{s} \times H^{s-1}}^{2}\right] e^{K_{4} T}-\beta,
\end{aligned}
$$

where $K_{4}$ is a fixed constant defined in (132).

Proof. From Lemma 18, we have $\inf _{x \in \mathbb{R}} u_{x}(t, q(t, x))=$ $\inf _{x \in \mathbb{R}} u_{x}(t, x)$ for all $t \in[0, T)$. Let $H(t, x)=u_{x}(t, q(t, x))+$ $\beta, \gamma(t, x)=\rho(t, q(t, x))$, and then

$$
\begin{aligned}
& \frac{\mathrm{d} H(t, x)}{\mathrm{d} t}=\left(u_{t x}+u u_{x x}\right)(t, q(t, x)), \\
& \frac{\mathrm{d} \gamma(t, x)}{\mathrm{d} t}=-\gamma(t, x)[H(t, x)-\beta] .
\end{aligned}
$$

Noting $m=0$ and using (90) yield

$$
\frac{\mathrm{d} H(t, x)}{\mathrm{d} t}=-\frac{1}{2} H^{2}+\frac{1}{2} \gamma^{2}+f(t, x)+\frac{1}{2} \beta^{2},
$$

where

$$
\begin{aligned}
f(t, x)= & {\left[u^{2}+2 k u-g *\left(u^{2}+\frac{u_{x}^{2}}{2}+2 k u+\frac{\rho^{2}}{2}\right)\right.} \\
& \left.-\lambda \partial_{x}\left(g * u^{2 n+1}\right)+\beta \partial_{x}(g * u)\right](t, q) .
\end{aligned}
$$

From the proof of Theorems 3 and 5, one deduces that

$$
\begin{aligned}
& |f(t, x)| \leq 3 C_{12}^{2}+1+(4 k+\beta) C_{12} \\
& +\frac{1}{2} \lambda\left(C_{12}\right)^{2 n+1}=K_{3}, \\
& \quad \text { for all }(t, x) \in[0, T) \times \mathbb{R} .
\end{aligned}
$$

For convenience, here we denote

$$
K_{4}=\max \left(K_{3}+\frac{1}{2}, \beta\right) .
$$

From (85), we obtain that $\gamma(t, x)$ has the same sign with $\gamma(0, x)=\rho_{0}(x)$ for all $x \in \mathbb{R}$. For $\eta_{0}(x) \in H^{s-1}$ with $s>3 / 2$, using Sobolev's embedding theorem, we have $\eta_{0}(x) \in C_{c}(\mathbb{R})$ 
and there exists a constant $\beta_{1}$ such that $\left|\eta_{0}(x)\right| \leq 1 / 2$ for all $|x| \geq \beta_{1}$. Since $\eta_{0}(x) \in C_{c}(\mathbb{R})$ and $\rho_{0}(x) \neq 0$ for all $x \in \mathbb{R}$, it follows that

$$
\inf _{|x| \leq \beta_{1}}|\gamma(0, x)|=\inf _{|x| \leq \beta_{1}}\left|\rho_{0}(x)\right|>0 .
$$

Taking $\beta_{2}=\min \left(1 / 2, \inf _{|x| \leq \beta_{1}}|\gamma(0, x)|\right)$, then $|\gamma(0, x)| \geq \beta_{2}>$ 0 for all $x \in \mathbb{R}$ and

$$
\gamma(t, x) \gamma(0, x)>0, \quad \text { for all } x \in \mathbb{R} .
$$

Now we consider the following Lyapunov function:

$$
\begin{array}{r}
w(t, x)=\gamma(0, x) \gamma(t, x)+\frac{\gamma(0, x)}{\gamma(t, x)}\left[1+H^{2}(t, x)\right], \\
(t, x) \in[0, T) \times \mathbb{R} .
\end{array}
$$

Applying Sobolev's embedding theorem yields

$$
\begin{aligned}
w(0, x) & =\gamma^{2}(0, x)+1+H^{2}(0, x) \\
& \leq 3+2 \beta^{2}+2\left\|\left(u_{0}, \rho_{0}-1\right)\right\|_{H^{s} \times H^{s-1}}^{2} .
\end{aligned}
$$

Differentiating (135) with respect to $t$ and using (129) and (132), we obtain

$$
\begin{aligned}
\frac{\mathrm{d} w(t, x)}{\mathrm{d} t}= & 2 \frac{\gamma(0, x)}{\gamma(t, x)} H(t, x)\left[\frac{1}{2}+f(t, x)\right] \\
& +\frac{\gamma(0, x)}{(\gamma(t, x))}\left(\gamma^{2}-1\right) \beta \\
\leq & \left(K_{3}+\frac{1}{2}\right) \frac{(\gamma(0, x))}{\gamma(t, x)}\left[1+H^{2}(t, x)\right] \\
& +\beta \gamma(0, x) \gamma(t, x) \\
\leq & K_{4} w(t, x) .
\end{aligned}
$$

By using Gronwall's inequality and (136), we deduce that

$$
\begin{aligned}
w(t, x) & \leq w(0, x) e^{K_{4} t} \\
& \leq\left(3+2 \beta^{2}+2\left\|u_{0}, \rho_{0}-1\right\|_{H^{s} \times H^{s-1}}^{2}\right) e^{K_{4} T},
\end{aligned}
$$

for all $(t, x) \in[0, T) \times \mathbb{R}$. On the other hand, from (135), we have

$$
\begin{aligned}
w(t, x) \geq & 2 \sqrt{\gamma^{2}(0, x)\left[1+H^{2}(t, x)\right]} \\
\geq & 2 \beta_{2}|H(t, x)|, \\
& \text { for all }(t, x) \in[0, T) \times \mathbb{R},
\end{aligned}
$$

which together with (138) yields that for all $(t, x) \in[0, T) \times \mathbb{R}$,

$$
\begin{aligned}
H(t, x) \geq & -\frac{1}{2 \beta_{2}} w(t, x) \geq-\frac{1}{2 \beta_{2}} \\
& \times\left[3+2 \beta^{2}+2\left\|\left(u_{0}, \rho_{0}-1\right)\right\|_{H^{s} \times H^{s-1}}^{2}\right] e^{K_{4} T} .
\end{aligned}
$$

Then, by the definition of $H(t, x)$, we complete the proof of Lemma 25.
Proof of Theorem 6. Combining the results of Theorem 1, (72) and Lemma 25, we complete the proof of Theorem 6.

Remark 26. Assume $m=0$ in system (1) and $\left(u_{0}, \rho_{0}-1\right) \epsilon$ $H^{s} \times H^{s-1}$ with $s>5 / 2$, and $\rho_{0}(x) \neq 0$ for all $x \in \mathbb{R}$. Let $T$ be the maximal existence time of the corresponding solution $(u, \rho-1)$ to system (1) with $m=0$, and then the corresponding solution blows up in finite time if and only if

$$
\lim _{t \rightarrow T} \sup \left\|\rho_{x}(t, \cdot)\right\|_{L^{\infty}}=+\infty
$$

Remark 27. For the same difficulty stated in Remark 21, here we only obtain the global existence result for solutions to system (1) with the assumption $m=0$.

\section{Conflict of Interests}

The authors declare that there is no conflict of interests regarding the publication of this paper.

\section{Acknowledgments}

The authors are grateful to the anonymous referees for a number of valuable comments and suggestions. This paper is supported by National Natural Science Foundation of China (71003082) and Fundamental Research Funds for the Central Universities (SWJTU12CX061 and SWJTU09ZT36).

\section{References}

[1] R. Camassa and D. D. Holm, "An integrable shallow water equation with peaked solitons," Physical Review Letters, vol. 71, no. 11, pp. 1661-1664, 1993.

[2] A. Constantin and D. Lannes, "The hydrodynamical relevance of the Camassa-Holm and Degasperis-Procesi equations," Archive for Rational Mechanics and Analysis, vol. 192, no. 1, pp. 165-186, 2009.

[3] A. Constantin, "The trajectories of particles in Stokes waves," Inventiones Mathematicae, vol. 166, no. 3, pp. 523-535, 2006.

[4] A. Constantin and J. Escher, "Particle trajectories in solitary water waves," Bulletin of the American Mathematical Society, vol. 44, no. 3, pp. 423-431, 2007.

[5] A. Constantin and W. A. Strauss, "Stability of peakons," Communications on Pure and Applied Mathematics, vol. 53, no. 5, pp. 603-610, 2000.

[6] A. Constantin and W. A. Strauss, "Stability of the CamassaHolm solitons," Journal of Nonlinear Science, vol. 12, no. 4, pp. 415-422, 2002.

[7] A. Constantin and W. A. Strauss, "Stability of a class of solitary waves in compressible elastic rods," Physics Letters A, vol. 270, no. 3-4, pp. 140-148, 2000.

[8] Y. A. Li and P. J. Olver, "Well-posedness and blow-up solutions for an integrable nonlinearly dispersive model wave equation," Journal of Differential Equations, vol. 162, no. 1, pp. 27-63, 2000.

[9] S. Lai and Y. Wu, "Local well-posedness and weak solutions for a weakly dissipative Camassa-Holm equation," Scientia Sinica Mathematica, vol. 40, no. 9, pp. 901-920, 2010.

[10] S. Lai and Y. Wu, "The local well-posedness and existence of weak solutions for a generalized Camassa-Holm equation," 
Journal of Differential Equations, vol. 248, no. 8, pp. 2038-2063, 2010.

[11] S. Lai and Y. Wu, "Global solutions and blow-up phenomena to a shallow water equation," Journal of Differential Equations, vol. 249, no. 3, pp. 693-706, 2010.

[12] S. Lai and Y. Wu, "A model containing both the Camassa-Holm and Degasperis-Procesi equations," Journal of Mathematical Analysis and Applications, vol. 374, no. 2, pp. 458-469, 2011.

[13] S. Lai and Y. Wu, "The study of global weak solutions for a generalized hyperelastic-rod wave equation," Nonlinear Analysis, vol. 80, pp. 96-108, 2013.

[14] C. Mu, S. Zhou, and R. Zeng, "Well-posedness and blow-up phenomena for a higher order shallow water equation," Journal of Differential Equations, vol. 251, no. 12, pp. 3488-3499, 2011.

[15] L. Tian, P. Zhang, and L. Xia, "Global existence for the higherorder Camassa-Holm shallow water equation," Nonlinear Analysis, vol. 74, no. 7, pp. 2468-2474, 2011.

[16] D.-X. Kong, "Global structure instability of Riemann solutions of quasilinear hyperbolic systems of conservation laws: rarefaction waves," Journal of Differential Equations, vol. 219, no. 2, pp. 421-450, 2005.

[17] Y. Guo, S. Lai, and Y. Wu, "On existence and uniqueness of the global weak solution for a shallow water equation," Applied Mathematics and Computation, vol. 218, no. 23, pp. 11410-11420, 2012.

[18] Y. Mi and C. Mu, "Well-posedness and analyticity for the Cauchy problem for the generalized Camassa-Holm equation," Journal of Mathematical Analysis and Applications, vol. 405, no. 1, pp. 173-182, 2013.

[19] S. Wu and Z. Yin, "Global existence and blow-up phenomena for the weakly dissipative Camassa-Holm equation," Journal of Differential Equations, vol. 246, no. 11, pp. 4309-4321, 2009.

[20] A. Constantin and R. I. Ivanov, "On an integrable 2-component Camassa-Holm shallow water system," Physics Letters A, vol. 372, no. 48, pp. 7129-7132, 2008.

[21] K. Yan and Z. Yin, "On the Cauchy problem for a 2-component Degasperis-Procesi system," Journal of Differential Equations, vol. 252, no. 3, pp. 2131-2159, 2012.

[22] K. Yan and Z. Yin, "Analytic solutions of the Cauchy problem for 2-component shallow water systems," Mathematische Zeitschrift, vol. 269, no. 3-4, pp. 1113-1127, 2011.

[23] C. Guan and Z. Yin, "Global existence and blow-up phenomena for an integrable 2-component Camassa-Holm shallow water system," Journal of Differential Equations, vol. 248, no. 8, pp. 2003-2014, 2010.

[24] C. Guan and Z. Yin, "Global weak solutions for a 2-component Camassa-Holm shallow water system," Journal of Functional Analysis, vol. 260, no. 4, pp. 1132-1154, 2011.

[25] J. Escher, O. Lechtenfeld, and Z. Yin, "Well-posedness and blowup phenomena for the 2-component Camassa-Holm equation," Discrete and Continuous Dynamical Systems, vol. 19, no. 3, pp. 493-513, 2007.

[26] G. Gui and Y. Liu, "On the global existence and wave-breaking criteria for the 2-component Camassa-Holm system," Journal of Functional Analysis, vol. 258, no. 12, pp. 4251-4278, 2010.

[27] G. Gui and Y. Liu, "On the Cauchy problem for the 2component camassa-Holm system," Mathematische Zeitschrift, vol. 268, no. 1-2, pp. 45-46, 2011.

[28] M. Chen, S.-Q. Liu, and Y. Zhang, "A 2-component generalization of the Camassa-Holm equation and its solutions," Letters in Mathematical Physics, vol. 75, no. 1, pp. 1-15, 2006.
[29] M. Zhu, "Blow-up, global existence and persistence properties for the coupled Camassa-Holm equations," Mathematical Physics, Analysis and Geometry, vol. 14, no. 3, pp. 197-209, 2011.

[30] Z. Guo and L. Ni, "Persistence properties and unique continuation of solutions to a 2-component Camassa-Holm equation," Mathematical Physics, Analysis and Geometry, vol. 14, no. 2, pp. 101-114, 2011.

[31] Y. Fu and C. Qu, "Unique continuation and persistence properties of solutions of the 2-component Degasperis-Procesi equations," Acta Mathematica Scientia, vol. 32, no. 2, pp. 652662, 2012.

[32] Y. Jin and Z. Jiang, "Wave breaking of an integrable CamassaHolm system with 2-components," Nonlinear Analysis, vol. 95, pp. 107-116, 2014.

[33] A. Shabat and L. M. Alonso, "On the prolongation of a hierarchy of hydrodynamic chains," in New Trends in Integrability and Partial Solvability, vol. 132 of NATO Science Series II, pp. 263280, Springer, Dordrecht, The Netherlands, 2004.

[34] L. Tian, W. Yan, and G. Gui, "On the local well posedness and blow-up solution of a coupled Camassa-Holm equations in Besov spaces," Journal of Mathematical Physics, vol. 53, Article ID 013701, 2012.

[35] W. Chen, X. Deng, and J. Zhang, "Blow up and blow-up rate for the generalized 2-component Camassa-Holm equation," International Journal of Nonlinear Science, vol. 12, no. 3, pp. 313322, 2011.

[36] W. Chen, L. Tian, X. Deng, and J. Zhang, "Wave breaking for a generalized weakly dissipative 2-component Camassa-Holm system," Journal of Mathematical Analysis and Applications, vol. 400, no. 2, pp. 406-417, 2013.

[37] C. Qu, Y. Fu, and Y. Liu, "Well-posedness, wave breaking and peakons for a modified $\mu$-Camassa-Holm equation," Journal of Functional Analysis, vol. 266, no. 2, pp. 433-477, 2013.

[38] Y. Fu, G. Gui, Y. Liu, and C. Qu, "On the Cauchy problem for the integrable modified Camassa-Holm equation with cubic nonlinearity," Journal of Differential Equations, vol. 255, no. 7, pp. 1905-1938, 2013.

[39] R. Danchin, "A few remarks on the Camassa-Holm equation," Differential and Integral Equations, vol. 14, no. 8, pp. 953-988, 2001.

[40] R. Danchin, Fourier Analysis Methods for PDEs, Lecture Notes, 2005.

[41] W. Yan, Y. Li, and Y. Zhang, "The Cauchy problem for the integrable Novikov equation," Journal of Differential Equations, vol. 253, no. 1, pp. 298-318, 2012.

[42] H. Bahouri, J.-Y. Chemin, and R. Danchin, Fourier Analysis and Nonlinear Partial Differential Equations, vol. 343 of Grundlehren der Mathematischen Wissenschafte, Springer, Berlin, Germany, 2011.

[43] Z. Yin, "On the Cauchy problem for an integrable equation with peakon solutions," Illinois Journal of Mathematics, vol. 47, no. 3, pp. 649-666, 2003.

[44] A. Constantin and J. Escher, "Wave breaking for nonlinear nonlocal shallow water equations," Acta Mathematica, vol. 181, no. 2, pp. 229-243, 1998.

[45] A. Constantin, "Existence of permanent and breaking waves for a shallow water equation: a geometric approach," Annales de l'Institut Fourier, vol. 50, no. 2, pp. 321-362, 2000. 


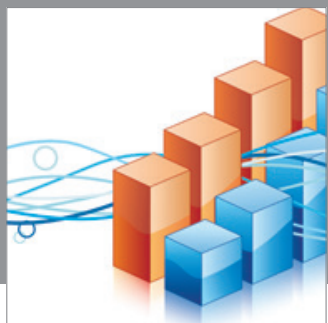

Advances in

Operations Research

mansans

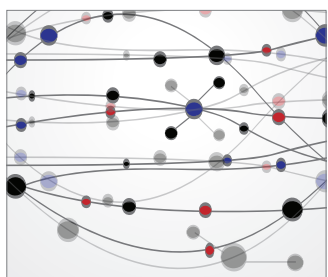

The Scientific World Journal
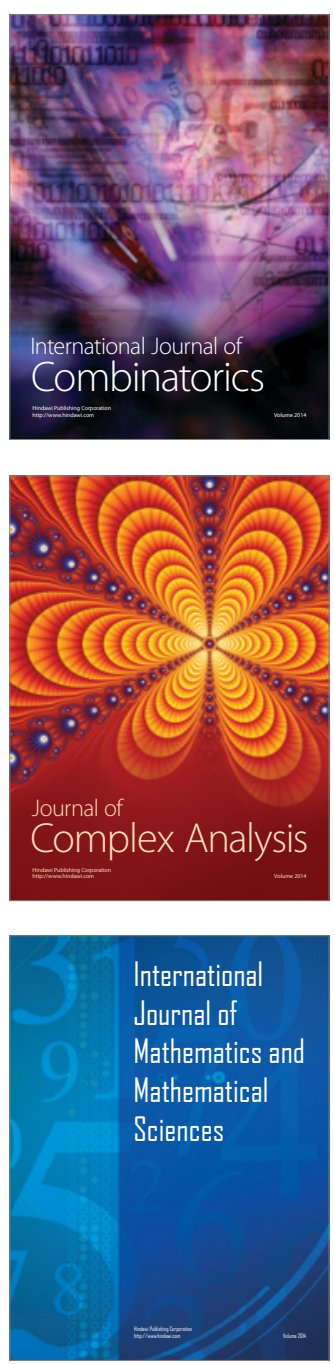
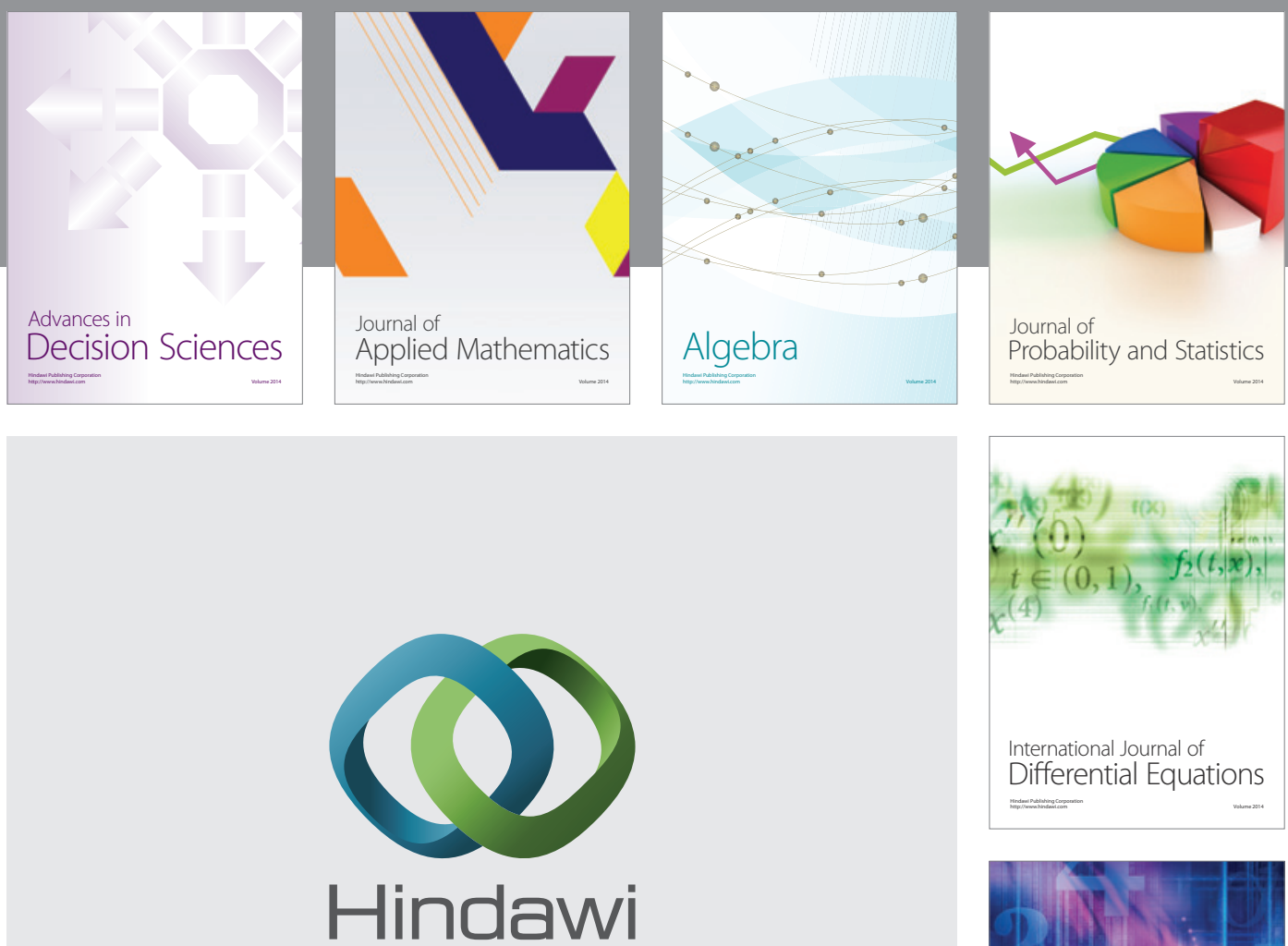

Submit your manuscripts at http://www.hindawi.com
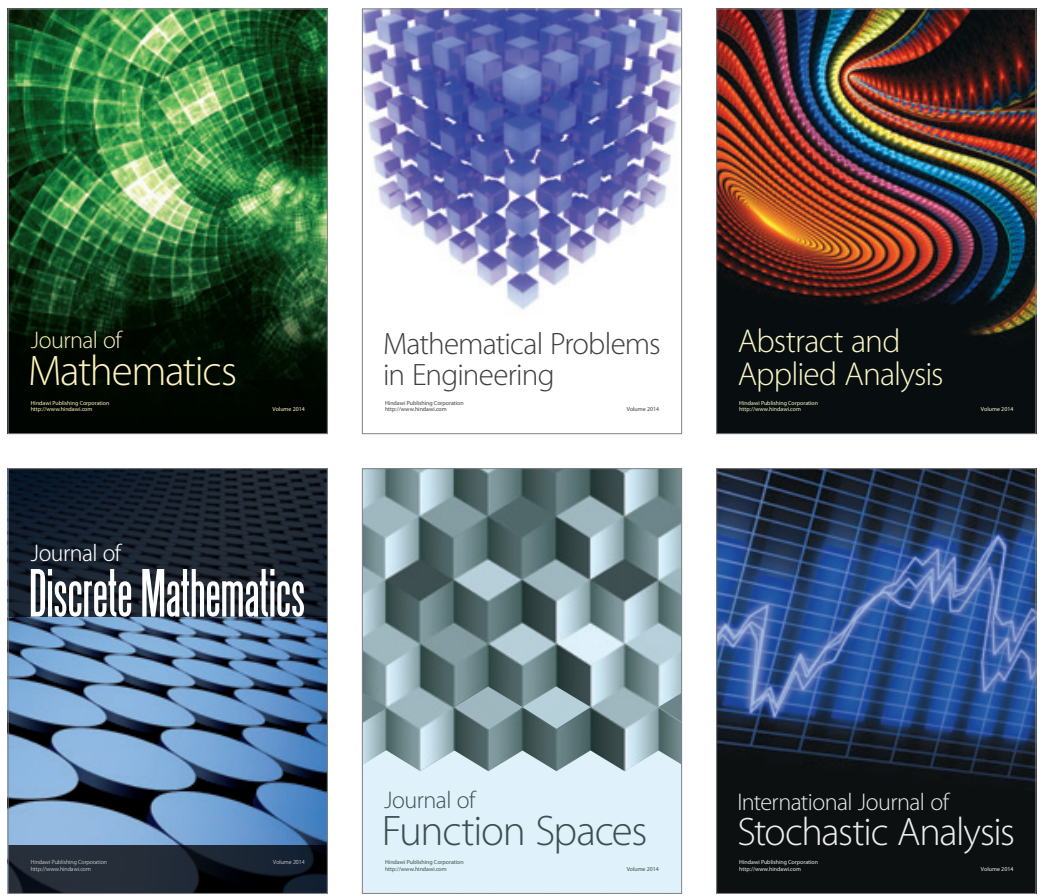

Journal of

Function Spaces

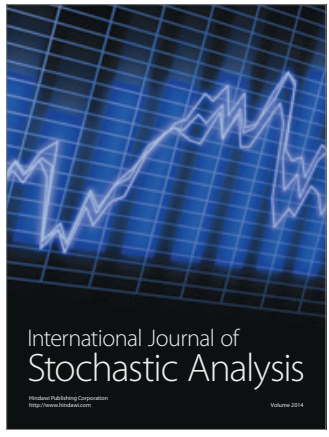

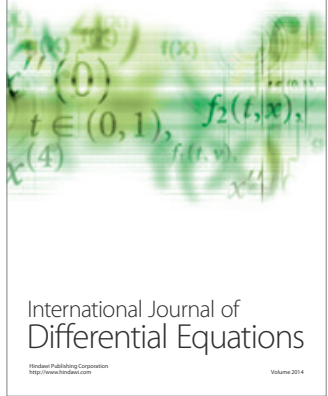
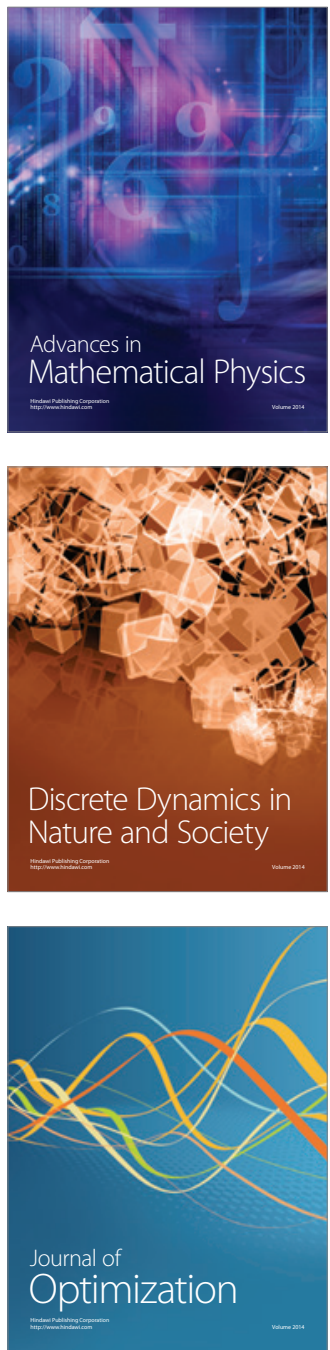